\title{
Strategy Guideline: Transitioning HVAC Companies to Whole House Performance Contractors
}

Arlan Burdick IBACOS 


\section{NOTICE}

This report was prepared as an account of work sponsored by an agency of the United States government. Neither the United States government nor any agency thereof, nor any of their employees, subcontractors, or affiliated partners makes any warranty, express or implied, or assumes any legal liability or responsibility for the accuracy, completeness, or usefulness of any information, apparatus, product, or process disclosed, or represents that its use would not infringe privately owned rights. Reference herein to any specific commercial product, process, or service by trade name, trademark, manufacturer, or otherwise does not necessarily constitute or imply its endorsement, recommendation, or favoring by the United States government or any agency thereof. The views and opinions of authors expressed herein do not necessarily state or reflect those of the United States government or any agency thereof.

Available electronically at http://www.osti.gov/bridge

Available for a processing fee to U.S. Department of Energy and its contractors, in paper, from:

U.S. Department of Energy

Office of Scientific and Technical Information

P.O. Box 62

Oak Ridge, TN 37831-0062

phone: 865.576 .8401

fax: 865.576 .5728

email: mailto:reports@adonis.osti.gov

Available for sale to the public, in paper, from:

U.S. Department of Commerce

National Technical Information Service

5285 Port Royal Road

Springfield, VA 22161

phone: 800.553 .6847

fax: 703.605.6900

email: orders@ntis.fedworld.gov

online ordering: http://www.ntis.gov/ordering.htm

Printed on paper containing at least $50 \%$ wastepaper, including $20 \%$ postconsumer waste 


\title{
Strategy Guideline: Transitioning HVAC Companies to Whole House Performance Contractors
}

\author{
Prepared for: \\ Building America \\ Building Technologies Program \\ Office of Energy Efficiency and Renewable Energy \\ U.S. Department of Energy \\ Prepared by: \\ Arlan Burdick \\ IBACOS, Inc. \\ 2214 Liberty Avenue \\ Pittsburgh, Pennsylvania 15222 \\ NREL Technical Monitor: Michael Gestwick \\ Prepared under Subcontract No. KNDJ-0-40341-02
}

May 2012 
[This page left blank] 


\section{Contents}

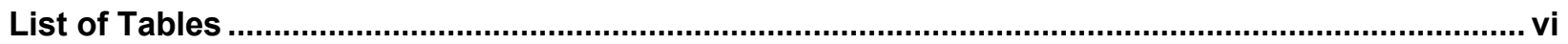

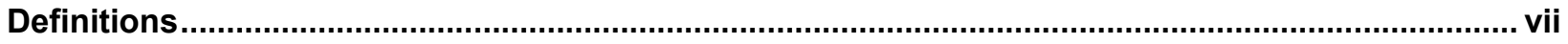

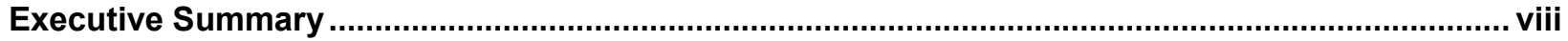

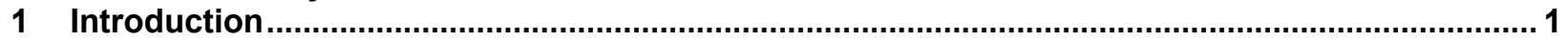

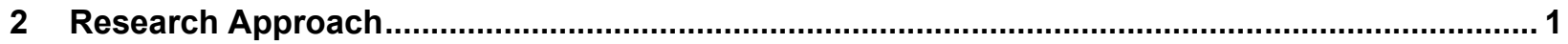

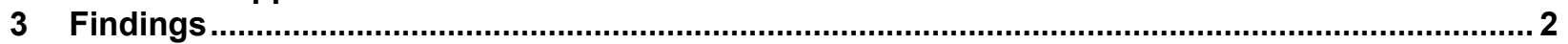

3.1 Literature Search Results ................................................................................... 2

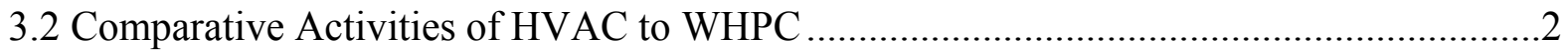

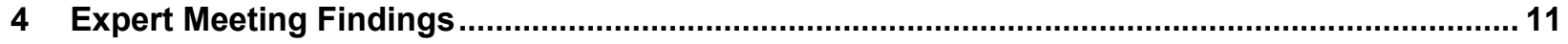

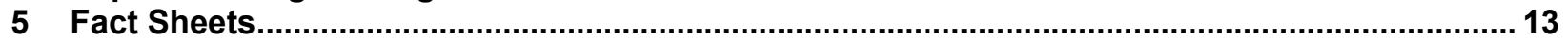

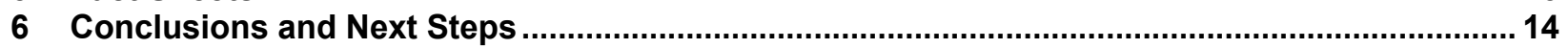

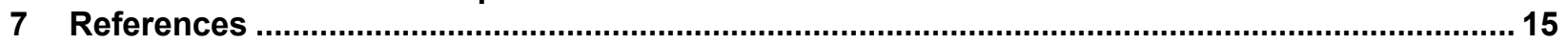

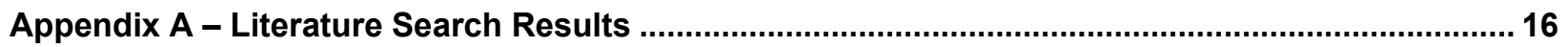

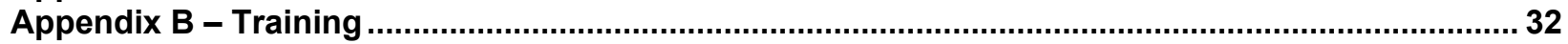

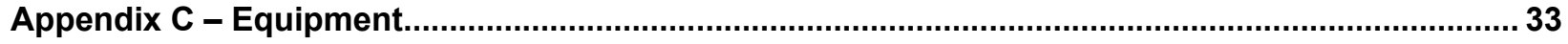

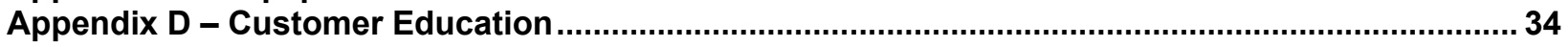

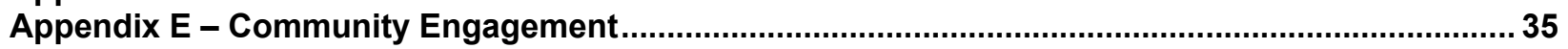

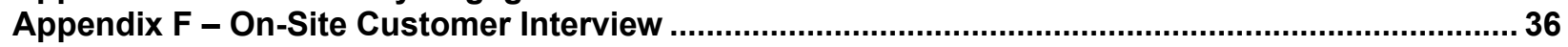

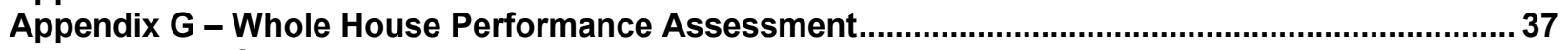

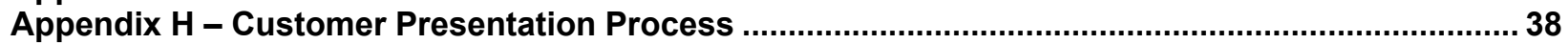

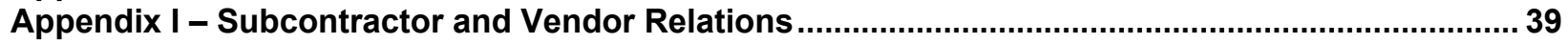

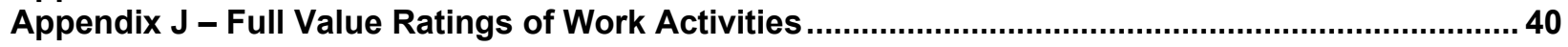




\section{List of Tables}

Table 1. Typical High-Level Business Operations Profile of a WHPC ............................................. 3

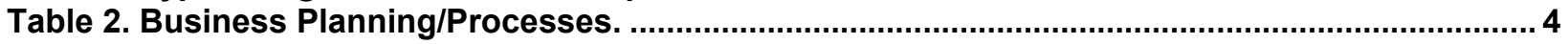

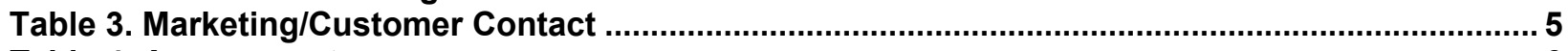

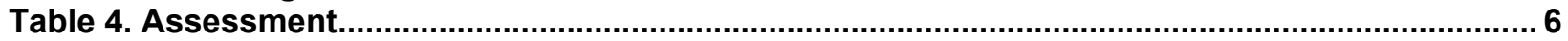

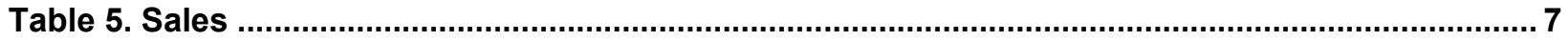

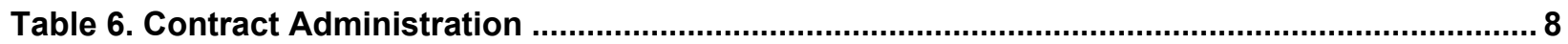

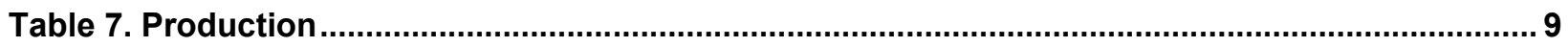

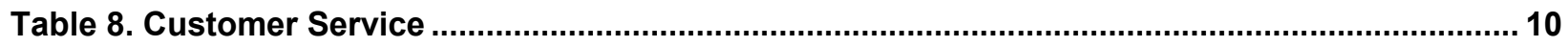

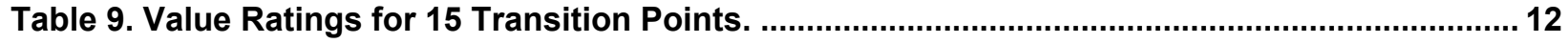

*Unless otherwise noted, all tables were created by IBACOS. 


\section{Definitions}

ACCA

HVAC

WHPC
Air Conditioning Contractors of America

heating, ventilation, and air conditioning

whole house performance contracting 


\section{Executive Summary}

This Strategy Guideline describes the findings from research that the U.S. Department of Energy's Building America team IBACOS conducted related to heating, ventilation, and air conditioning (HVAC) companies that have made the decision to transition to whole house performance contracting (WHPC). By utilizing a house-as-a-system approach, the WHPC contractor can evaluate the total energy performance of the entire house and make upgrades on a prioritized basis, according to the energy benefit.

IBACOS believes that an HVAC contractor is well suited to take on the tasks of whole house energy upgrades and to advance large-scale energy efficiency in the residential market. Because the HVAC contractor already works on one of the most technically complex systems in the house, they have already overcome the barrier of training to the complex HVAC system and can reduce the time required to transition to delivering whole house energy upgrades on a wide scale. Providing contractors with new technical skills and a revised business approach is one way to achieve change on a large scale and to help the United States meet its goals regarding job creation and energy independence. However, HVAC companies need guidance on how to transition successfully from their traditional business approach to one that includes WHPC.

Research for this Strategy Guideline included a literature search of periodicals, industry trade publications, and reports available to the general public on the topic of WHPC. IBACOS created an outline of a successfully integrated HVAC company with a WHPC business and presented the outline to a group of experts currently working in the industry. The feedback received from the experts was used to identify the key areas in which guidance would be most useful to WHPC contractors, and eight of these areas were expanded for guidance.

The appendices included with this Strategy Guideline present information a transitioning contractor may find useful and were collected from published periodicals, along with short and concise Fact Sheets. 


\section{Introduction}

This Strategy Guideline describes the findings from research IBACOS conducted related to heating, ventilation, and air conditioning (HVAC) companies who have made the decision to transition to whole house performance contracting (WHPC). Home performance is the energyrelated services offered by a contractor who uses testing and building science to diagnose and repair issues of comfort, safety, durability, and energy efficiency in a house. Whole house performance contracting brings control of the home performance tasks of improving the insulation, HVAC, air sealing, windows, and appliances in a house under one company. Integrating the necessary energy upgrades allows for the analysis of the comfort, energy efficiency, and cost of the upgrades so the highest-value energy upgrades can be completed first.

IBACOS believes that an HVAC contractor is well suited to take on the tasks of whole house energy upgrades and to advance large-scale energy efficiency in the residential market. HVAC companies were the focus of this study, based on their market position of typically having an existing and ongoing relationship with homeowners through service and maintenance contracts. This ongoing relationship places the HVAC contractor in an ideal position to deliver energy upgrades to whole communities or sections of communities. The HVAC contractor already works on one of the most technically complex systems in the house, eliminating the barrier of training to the complex HVAC system and reducing the time required to transition to delivering whole house energy upgrades on a wide scale. Energy upgrades may be supplemental to an HVAC contractor's core business, a new guiding strategy for their company, or a sales tool to enable up-selling to a larger project in partnership with other qualified trade contractors. Providing contractors with new technical skills and a revised business approach is one way to achieve change on a large scale and to help the United States meet its goals regarding job creation and energy independence.

There are two primary ways to define the routes of transition for HVAC companies taking on WHPC: (1) subcontracting out the shell repair and upgrade work or (2) integrating the shell repair and upgrade work into their existing businesses.

The appendices included with this Strategy Guideline present the information a transitioning contractor may find useful from published periodicals, along with short and concise Fact Sheets.

\section{Research Approach}

IBACOS defined the following research questions relative to this area of study:

- What are the business best practices associated with well-run WHPC companies?

- What are the business profiles for HVAC companies to offer WHPC?

- What are the transition strategies for HVAC companies who want to become home performance contractors?

This research was not intended to identify motivation for HVAC companies to make the transition to WHPC, but rather to identify the topics where HVAC companies will need guidance on how to successfully transition from their traditional business to one that includes WHPC. 
Research began with a literature search of periodicals, industry trade publications, and reports available to the general public on the topic of WHPC. Individuals currently involved in home performance work and HVAC companies who have brought WHPC into their service offerings were interviewed. From the information gained through the literature search and individual interviews, IBACOS created an outline of a successfully integrated HVAC and WHPC business. Work activities that represent areas of transition for the HVAC companies were identified. An Expert Meeting (IBACOS 2011) was held, where industry participants were asked to validate completeness of the outline and to rate the identified transition points. The highest rated transition points from the Expert Meeting have been extracted for further study and comment in the appendices of this Strategy Guideline.

\section{Findings}

\subsection{Literature Search Results}

The literature search spanned back across 10 years to the late 1990s, not because the search was limited to that time frame, but because home performance began to emerge as an industry during that time. As a tool for the transitioning contractor, a categorized bibliography of pertinent articles from that literature search is included as Appendix A in this Strategy Guideline.

Home Energy magazine, published by Energy Auditor \& Retrofitter, Inc., was an obvious place to begin the search because the mission of that publication is to disseminate objective and practical information on residential energy efficiency, performance, comfort, and affordability. A Hanley Wood LLC publication, The Journal of Light Construction, written for residential and light commercial contractors, also included articles on energy upgrade topics. From the perspective of the HVAC industry, home performance has been the topic of an annual special edition of the Air Conditioning Contractors of America (ACCA) monthly periodical, Contractor Excellence, since 2004. Periodicals offer a continuing resource of current information to the transitioning contractor because they tend to track recent developments within the industry.

Reports from previous studies found in the search included a Bevilacqua Knight report titled "PIER Whole House Contracting Study and Charting the Home Performance Contractors Territory" (Bevilacqua Knight November 2003). A conclusion in that study stated that, based on their survey results at that time, "Surprisingly, HVAC contractors, who typically have greater technical expertise, appeared much less likely to embrace whole house approaches that integrate shell with HVAC disciplines."

\subsection{Comparative Activities of HVAC to WHPC}

Table 1 shows a high-level outline of how an integrated WHPC company could be divided into seven Operational Areas, based on the information gained from the literature search of periodicals, industry trade publications, reports, and interviews performed by IBACOS with industry experts. Shaded cells in the Business Functions rows identify areas where noted differences exist between the HVAC contractor's typical Business Function and the WHPC Business Function. 
Table 1. Typical High-Level Business Operations Profile of a WHPC*

\begin{tabular}{|c|c|c|c|c|c|c|c|}
\hline $\begin{array}{l}\text { Operational } \\
\text { Area }\end{array}$ & $\begin{array}{l}\text { Business } \\
\text { Planning/ } \\
\text { Processes }\end{array}$ & $\begin{array}{l}\text { Marketing/ } \\
\text { Customer } \\
\text { Contact }\end{array}$ & Assessment & Sales & $\begin{array}{l}\text { Contract } \\
\text { Administration }\end{array}$ & Production & $\begin{array}{l}\text { Customer } \\
\text { Service }\end{array}$ \\
\hline \multirow[t]{5}{*}{$\begin{array}{l}\text { Business } \\
\text { Functions }\end{array}$} & Training & PR Strategies & $\begin{array}{l}\text { Customer } \\
\text { Interaction }\end{array}$ & Proposal & $\begin{array}{l}\text { Contract } \\
\text { Processing }\end{array}$ & $\begin{array}{l}\text { Project Planning } \\
\text { and Scheduling }\end{array}$ & $\begin{array}{l}\text { Customer } \\
\text { Relations }\end{array}$ \\
\hline & $\begin{array}{l}\text { Employee } \\
\text { Relations }\end{array}$ & $\begin{array}{l}\text { Advertising } \\
\text { Strategies }\end{array}$ & $\begin{array}{l}\text { Whole House } \\
\text { Performance } \\
\text { Assessment }\end{array}$ & $\begin{array}{l}\text { Sales } \\
\text { Presentation to } \\
\text { Customer }\end{array}$ & $\begin{array}{l}\text { Customer } \\
\text { Financing }\end{array}$ & $\begin{array}{l}\text { Project } \\
\text { Implementation }\end{array}$ & $\begin{array}{l}\text { Warranty } \\
\text { Requests } \\
\text { Resolution }\end{array}$ \\
\hline & Procurement & $\begin{array}{l}\text { Customer } \\
\text { Referral } \\
\text { Strategies }\end{array}$ & & Closing & $\begin{array}{l}\text { Rebates and } \\
\text { Incentives }\end{array}$ & $\begin{array}{l}\text { Quality } \\
\text { Assurance }\end{array}$ & \\
\hline & Subcontracts & $\begin{array}{l}\text { Call } \\
\text { Management }\end{array}$ & & & \begin{tabular}{|l|} 
Accounts \\
Receivable/ \\
Payable
\end{tabular} & & \\
\hline & $\begin{array}{l}\text { Strategic } \\
\text { Planning }\end{array}$ & $\begin{array}{l}\text { Lead } \\
\text { Management }\end{array}$ & & & & & \\
\hline
\end{tabular}

* Based on research, an integrated WHPC company could be divided into these seven Operational Areas. Shaded cells in the Business Functions rows identify areas where noted differences exist between the HVAC contractor's typical Business Function and the WHPC Business Function. 
Tables 2 through 8 further break down the Business Functions into Work Activities. The Work Activities where a transitioning HVAC contractor is anticipated to need guidance are shaded in yellow in these tables and are referred to as Transition Points in the remainder of this guideline.

Table 2. Business Planning/Processes*

\begin{tabular}{|c|c|c|c|c|c|}
\hline \multirow{2}{*}{\begin{tabular}{|l|}
$\begin{array}{l}\text { Operational } \\
\text { Area }\end{array}$ \\
$\begin{array}{l}\text { Business } \\
\text { Function }\end{array}$ \\
\end{tabular}} & \multicolumn{5}{|c|}{ Business Planning/Processes } \\
\hline & Training & $\begin{array}{l}\text { Employee } \\
\text { Relations }\end{array}$ & Procurement & Subcontracts & \begin{tabular}{|l} 
Strategic \\
Planning
\end{tabular} \\
\hline \multirow[t]{5}{*}{ Work Activities } & Technical & $\begin{array}{l}\text { Job } \\
\text { Progression/ } \\
\text { Growth }\end{array}$ & \begin{tabular}{|l} 
Manufacturer \\
Relations
\end{tabular} & $\begin{array}{l}\text { Vendor } \\
\text { Relations }\end{array}$ & \begin{tabular}{|l} 
Company \\
Mission/Vision
\end{tabular} \\
\hline & Safety & Retention & $\begin{array}{l}\text { Material } \\
\text { Inventory }\end{array}$ & Pricing & $\begin{array}{l}\text { Five-Year } \\
\text { Strategic Plan }\end{array}$ \\
\hline & Management & Compensation & New Equipment & & $\begin{array}{l}\text { One-Year } \\
\text { Operational } \\
\text { Plan }\end{array}$ \\
\hline & Sales & Referral Bonus & $\begin{array}{l}\text { Equipment } \\
\text { Maintenance/ } \\
\text { Repair/ } \\
\text { Calibration }\end{array}$ & & $\begin{array}{l}\text { Management } \\
\text { Objectives }\end{array}$ \\
\hline & & $\begin{array}{l}\text { Quality } \\
\text { Management }\end{array}$ & Pricing & & $\begin{array}{l}\text { Quality } \\
\text { Management }\end{array}$ \\
\hline Internal Lead & $\begin{array}{l}\text { Leadership } \\
\text { Team }\end{array}$ & $\begin{array}{l}\text { Leadership } \\
\text { Team }\end{array}$ & $\begin{array}{l}\text { Leadership } \\
\text { Team }\end{array}$ & $\begin{array}{l}\text { Leadership } \\
\text { Team }\end{array}$ & $\begin{array}{l}\text { Leadership } \\
\text { Team }\end{array}$ \\
\hline $\begin{array}{l}\text { External } \\
\text { Resources }\end{array}$ & \begin{tabular}{|l|} 
Training/ \\
Certification \\
Providers
\end{tabular} & $\begin{array}{l}\text { HR/Quality } \\
\text { Management } \\
\text { Consultants }\end{array}$ & Manufacturers & $\begin{array}{l}\text { Subcontractors, } \\
\text { Manufacturers }\end{array}$ & $\begin{array}{l}\text { Management } \\
\text { Consultants }\end{array}$ \\
\hline
\end{tabular}

* Highlighted cells indicate Work Activities that are Transition Points.

In Table 2, the highlighted cells indicate Work Activities that are Transition Points. The Work Activities identified as Transition Points for the Business Planning/Processes area are as follows:

- Technical Training

- Manufacturer Relations

- New Equipment

- Equipment Maintenance/Repair/Calibration

- Vendor Relations

- Company Vision/Mission

- Five-Year Strategic Plan

- One-Year Operational Plan

The areas of Technical Training and Equipment Maintenance/Repair/Calibration requirements are expanded further in Appendix B and Appendix C, respectively, in this Strategy Guideline. 
Table 3. Marketing/Customer Contact*

\begin{tabular}{|c|c|c|c|c|c|}
\hline \multirow{2}{*}{$\begin{array}{l}\text { Operational } \\
\text { Area } \\
\text { Business } \\
\text { Function }\end{array}$} & \multicolumn{5}{|c|}{ Marketing/Customer Contact } \\
\hline & PR Strategies & $\begin{array}{l}\text { Advertising } \\
\text { Strategies }\end{array}$ & \begin{tabular}{|l|} 
Customer \\
Referral \\
Strategies
\end{tabular} & \begin{tabular}{|l|} 
Call \\
Management
\end{tabular} & $\begin{array}{l}\text { Lead } \\
\text { Management }\end{array}$ \\
\hline \multirow[t]{6}{*}{ Work Activities } & Newspaper & Newspaper & $\begin{array}{l}\text { Community } \\
\text { Engagement }\end{array}$ & Call Scripting & $\begin{array}{l}\text { Sales } \\
\text { Coordination }\end{array}$ \\
\hline & Local TV/Radio & Local TV/Radio & Social Media & $\begin{array}{l}\text { Inbound Call } \\
\text { Process }\end{array}$ & $\begin{array}{l}\text { Lead Database } \\
\text { and Tracking }\end{array}$ \\
\hline & Local Expert & Billboards & \begin{tabular}{|l|} 
Direct Referral \\
Programs
\end{tabular} & $\begin{array}{l}\text { Recurring Call } \\
\text { Schedule }\end{array}$ & $\begin{array}{l}\text { Lead } \\
\text { Qualification }\end{array}$ \\
\hline & \begin{tabular}{|l|} 
Customer \\
Education
\end{tabular} & \begin{tabular}{|l|} 
Neighborhood \\
Blitz \\
\end{tabular} & \begin{tabular}{|l|} 
Newsletter \\
(E- or Paper)
\end{tabular} & & Scheduling \\
\hline & Home Shows & Web Site & \begin{tabular}{|l|} 
Service Tech \\
Referrals
\end{tabular} & & $\begin{array}{l}\text { Appointment } \\
\text { Verification }\end{array}$ \\
\hline & & $\begin{array}{l}\text { Search Engine } \\
\text { Optimization }\end{array}$ & & & \\
\hline Internal Lead & Marketing & Marketing & Marketing & Operations & $\begin{array}{l}\text { Sales/ } \\
\text { Operations }\end{array}$ \\
\hline $\begin{array}{l}\text { External } \\
\text { Resources }\end{array}$ & PR Agency & $\begin{array}{l}\text { Ad Agency/ } \\
\text { Designer }\end{array}$ & $\begin{array}{l}\text { Ad Agency/ } \\
\text { Designer/ } \\
\text { Community } \\
\text { Groups }\end{array}$ & \begin{tabular}{|l} 
Sales \\
Consultant
\end{tabular} & \begin{tabular}{|l|} 
Sales \\
Consultant
\end{tabular} \\
\hline
\end{tabular}

* Highlighted cells indicate Work Activities that are Transition Points.

In Table 3, the highlighted cells indicate Work Activities that are Transition Points. The Work Activities identified as Transition Points for the Marketing/Customer Contact area are as follows:

- Customer Education

- Community Engagement

- Social Media

- Direct Referral Programs

- Newsletter

- $\quad$ Service Tech Referrals

- Call Scripting

- Inbound Call Process

- Recurring Call Schedule 
The areas of Customer Education and Community Engagement are expanded further in Appendix D and Appendix E, respectively, in this Strategy Guideline.

Table 4. Assessment*

\begin{tabular}{|l|l|l|}
\hline $\begin{array}{l}\text { Operational } \\
\text { Area }\end{array}$ & Assessment & \multicolumn{2}{|l|}{} \\
\hline $\begin{array}{l}\text { Business } \\
\text { Function }\end{array}$ & Customer Interaction & $\begin{array}{l}\text { Whole House Performance } \\
\text { Assessment }\end{array}$ \\
\hline Work Activities & Pre-Assessment Preparation Call & $\begin{array}{l}\text { Exterior Architectural Features } \\
\text { Assessment }\end{array}$ \\
\cline { 2 - 3 } & Arrival Process & Interior Building Features Assessment \\
\cline { 2 - 3 } & On-Site Customer Interview & Thermal Enclosure Components \\
\cline { 2 - 3 } & & Space Conditioning System Assessment \\
\cline { 2 - 3 } & & Plumbing System Assessment \\
\cline { 2 - 3 } & & Electrical System Assessment \\
\cline { 2 - 3 } & & Moisture Assessment \\
\cline { 2 - 3 } & & System Testing \\
\cline { 2 - 3 } & & Operations \\
\hline External & & \\
\hline
\end{tabular}

* Highlighted cells indicate Work Activities that are Transition Points.

In Table 4, the highlighted cells indicate Work Activities that are Transition Points. The Work Activities identified as Transition Points for the Assessment area are as follows:

- Pre-Assessment Preparation

- Arrival Process

- On-Site Customer Interview

- Whole House Performance Assessment

Whole House Performance Assessment was identified as the area with many new facets of the service offering for the HVAC contractor transitioning to WHPC. The areas of On-Site Customer Interview and Whole House Performance Assessment are expanded further in Appendix F and Appendix G, respectively, in this Strategy Guideline. 
Table 5. Sales*

\begin{tabular}{|l|l|l|l|}
\hline $\begin{array}{l}\text { Operational } \\
\text { Area }\end{array}$ & \multicolumn{2}{|l|}{ Sales } \\
\hline $\begin{array}{l}\text { Business } \\
\text { Function }\end{array}$ & Proposal & $\begin{array}{l}\text { Sales Presentation to } \\
\text { Customer }\end{array}$ & Closing \\
\hline \multirow{4}{*}{ Work Activities } & $\begin{array}{l}\text { Work Scope Development } \\
\text { and Approval }\end{array}$ & Close at Audit Process & Contracting \\
\cline { 2 - 4 } & $\begin{array}{l}\text { Customer Presentation } \\
\text { Process }\end{array}$ & Follow-Up Sales Process & Identify Financing Needs \\
\cline { 2 - 4 } & Anticipated Objections & $\begin{array}{l}\text { Phased Implementation } \\
\text { Sales Process }\end{array}$ & Rebates and Incentives \\
\cline { 2 - 4 } & Assessment Report & Overcoming Objections & \\
\cline { 2 - 4 } & Pricing & & Sales/Operations \\
\cline { 2 - 4 } & Subcontracts & Sales & Finance Partners \\
\hline Internal Lead & Sales & & \\
\hline $\begin{array}{l}\text { External } \\
\text { Resources }\end{array}$ & Subcontractors & & \\
\hline
\end{tabular}

* Highlighted cells indicate Work Activities that are Transition Points.

In Table 5, the highlighted cells indicate Work Activities that are Transition Points. The Work Activities identified as Transition Points for the Sales area are as follows:

- Customer Presentation Process

- Assessment Report

- Phased Implementation Sales Process

- Rebates and Incentives

The area of Customer Presentation Process is expanded further in Appendix $\mathrm{H}$ in this Strategy Guideline. 
Table 6. Contract Administration*

\begin{tabular}{|l|l|l|l|l|}
\hline $\begin{array}{l}\text { Operational } \\
\text { Area }\end{array}$ & \multicolumn{3}{|l|}{ Contract Administration } \\
\hline $\begin{array}{l}\text { Business } \\
\text { Function }\end{array}$ & $\begin{array}{l}\text { Contract } \\
\text { Processing }\end{array}$ & $\begin{array}{l}\text { Customer } \\
\text { Financing }\end{array}$ & $\begin{array}{l}\text { Rebates and } \\
\text { Incentives }\end{array}$ & $\begin{array}{l}\text { Accounts } \\
\text { Receivable/ } \\
\text { Payable }\end{array}$ \\
\hline \multirow{4}{*}{ Work Activities } & $\begin{array}{l}\text { Contract Review } \\
\text { and Approval }\end{array}$ & $\begin{array}{l}\text { Financing } \\
\text { Mechanisms }\end{array}$ & Utility Reporting & Create Account \\
\cline { 2 - 5 } & Create Work Order & Loan Processing & $\begin{array}{l}\text { State or Local } \\
\text { Program Reporting }\end{array}$ & Deposit Payments \\
\cline { 2 - 5 } & $\begin{array}{l}\text { Inventory and } \\
\text { Production } \\
\text { Management }\end{array}$ & & $\begin{array}{l}\text { Tax Credit } \\
\text { Reporting }\end{array}$ & Billing \\
\cline { 2 - 5 } & Subcontracts & & Payment Terms \\
\hline Internal Lead & Operations & Operations & Operations & Operations \\
\hline $\begin{array}{l}\text { External } \\
\text { Resources }\end{array}$ & Subcontractors & Financial Partners & Program Staff & \\
\hline
\end{tabular}

* Highlighted cells indicate Work Activities that are Transition Points.

In Table 6, the highlighted cells indicate Work Activities that are Transition Points. The Work Activities identified as Transition Points for the Contract Administration area are as follows:

- Financing Mechanisms

- Loan Processing

- Utility Reporting

- State or Local Program Reporting

Although these are all important Work Activities, they are not expanded further in this Strategy Guideline due to the lack of available technical material related to these Transition Points. 
Table 7. Production*

\begin{tabular}{|l|l|l|l|}
\hline $\begin{array}{l}\text { Operational } \\
\text { Area }\end{array}$ & \multicolumn{2}{|l|}{ Production } \\
\hline $\begin{array}{l}\text { Business } \\
\text { Function }\end{array}$ & $\begin{array}{l}\text { Project Planning and } \\
\text { Scheduling }\end{array}$ & Project Implementation & Quality Assurance \\
\hline \multirow{4}{*}{ Work Activities } & Scheduling & Work Scope Procedures & Job Completion Verification \\
\cline { 2 - 4 } & $\begin{array}{l}\text { Inventory/Material } \\
\text { Preparation }\end{array}$ & Job Safety & $\begin{array}{l}\text { Post-Installation Field } \\
\text { Verification Sample }\end{array}$ \\
\cline { 2 - 4 } & $\begin{array}{l}\text { Crew Lead Work Scope } \\
\text { Review }\end{array}$ & $\begin{array}{l}\text { Customer Interaction } \\
\text { Procedures }\end{array}$ & $\begin{array}{l}\text { Customer Satisfaction } \\
\text { Scores }\end{array}$ \\
\cline { 2 - 4 } & & Job Completion/Test Out & \\
\cline { 2 - 4 } & & Change Orders & $\begin{array}{l}\text { Sponsoring Program } \\
\text { Quality Assurance }\end{array}$ \\
\cline { 2 - 5 } & & $\begin{array}{l}\text { Subcontractor and Vendor } \\
\text { Relations }\end{array}$ & \\
\hline Internal Lead & Operations/Production & Production & Operations/Production \\
\hline $\begin{array}{l}\text { External } \\
\text { Resources }\end{array}$ & & & \\
\hline
\end{tabular}

* Highlighted cells indicate Work Activities that are Transition Points.

In Table 7, the highlighted cells indicate Work Activities that are Transition Points. The Work Activities identified as Transition Points for the Production area are as follows:

- Work Scope Procedures

- Customer Interaction Procedures

- Job Completion/Test Out

- Job Completion Verification

- Post-Installation Field Verification Sample

- Sponsoring Program Quality Assurance

- Subcontractor and Vendor Relations

The area of Subcontractor and Vendor Relations is expanded further in Appendix I in this Strategy Guideline. 
Table 8. Customer Service*

\begin{tabular}{|l|l|l|}
\hline $\begin{array}{l}\text { Operational } \\
\text { Area }\end{array}$ & Customer Service \\
\hline $\begin{array}{l}\text { Business } \\
\text { Function }\end{array}$ & Customer Relations & Warranty Requests Resolution \\
\hline \multirow{4}{*}{ Work Activities } & Customer Appreciation & \\
\cline { 2 - 3 } & Certificate & Customer Care Intake \\
\cline { 2 - 3 } & Rating Score & Scheduling \\
\cline { 2 - 3 } & Guarantee & Customer Visit Process \\
\cline { 2 - 3 } & Homeowner Manual & Utility Bill Review \\
\cline { 2 - 3 } & Referral Cards/Lead Generation & Service Call Diagnostics \\
\cline { 2 - 3 } & Billing and Collections & Warrantable Item Repair/Replacement \\
\cline { 2 - 3 } & Utility/Local Program Documentation & Subcontractor Management \\
\hline Internal Lead & Operations & Production \\
\hline $\begin{array}{l}\text { External } \\
\text { Resources }\end{array}$ & & \\
\hline
\end{tabular}

* Highlighted cells indicate Work Activities that are Transition Points.

In Table 8, the highlighted cells indicate Work Activities that are Transition Points. The Work Activities identified as Transition Points for the Customer Service area are as follows:

- Utility Bill Review

- Service Call Diagnostics

- Warrantable Item Repair/Replacement

Although these are all important Work Activities, they are not expanded further in this Strategy Guideline due to the lack of available technical material related to these Transition Points. 


\section{Expert Meeting Findings}

The outline of a successfully integrated HVAC and WHPC business was presented to a group of industry experts at an Expert Meeting. Details of this Expert Meeting have been published in the Building America report, "Final Expert Meeting Report: Transitioning Traditional HVAC Contractors to Whole House Performance Contractors" (IBACOS 2011).

At this Expert Meeting, the panel was asked to validate the accuracy and the comprehensiveness of the outline in capturing the business activities. The expert panel praised the level of detail to which the outline was broken out and validated its accuracy. Transition Points were validated and carefully rated for their value of guidance and difficulty to implement. The ratings of the Transition Points were valued where " 1 " is a low value to the transitioning contractor through " 4 " being the highest value to the transitioning contractor, as well as " 1 " being the easiest to execute through " 4 " being the most difficult to execute. The combined score of value and ease of execution was used to rank the importance for further study.

There were 15 Transition Points that rose to the top as being rated " 4 " (highest) for value to the transitioning contractor. Table 9 shows the scores for each of these 15 Transition Points. The entire matrix of value to a transitioning contractor and difficulty to implement, as determined by the expert panel, can be found in Appendix J of this Strategy Guideline.

- Technical Training

- Equipment Requirements

- Vendor Relations

- Strategic Planning

- Customer Education Process

- Community Engagement

- Assessment Arrival Process

- On-Site Customer Interview

- Whole House Performance Assessment

- Customer Presentation Process

- Assessment Report

- State or Local Program Reporting

- Work Scope/Procedures

- Job Completion Verification

- Subcontractor Relations 
Table 9. Value Ratings for 15 Transition Points

\begin{tabular}{|c|c|c|c|c|}
\hline Operational Area & Work Activity & \begin{tabular}{|l} 
Value of \\
Guidance to \\
Contractor
\end{tabular} & Difficulty & Sum \\
\hline $\begin{array}{l}\text { Business Planning/ } \\
\text { Process }\end{array}$ & Training & 4 & 2 & 6 \\
\hline $\begin{array}{l}\text { Business Planning/ } \\
\text { Process }\end{array}$ & Equipment & 4 & 3 & 7 \\
\hline $\begin{array}{l}\text { Business Planning/ } \\
\text { Process }\end{array}$ & Vendor Relations & 4 & 4 & 8 \\
\hline $\begin{array}{l}\text { Business Planning/ } \\
\text { Process }\end{array}$ & Strategic Planning & 4 & 4 & 8 \\
\hline $\begin{array}{l}\text { Marketing/ } \\
\text { Customer Contact }\end{array}$ & Customer Education & 4 & 4 & 8 \\
\hline $\begin{array}{l}\text { Marketing/ } \\
\text { Customer Contact }\end{array}$ & Community Engagement & 4 & 4 & 8 \\
\hline Assessment & Arrival Process & 4 & 3 & 7 \\
\hline Assessment & On-Site Customer Interview & 4 & 4 & 8 \\
\hline Assessment & $\begin{array}{l}\text { Whole House Performance } \\
\text { Assessment }\end{array}$ & 4 & 4 & 8 \\
\hline Sales & $\begin{array}{l}\text { Customer Presentation } \\
\text { Process }\end{array}$ & 4 & 3 & 7 \\
\hline Sales & Assessment Report & 4 & 3 & 7 \\
\hline Contract Administration & $\begin{array}{l}\text { State or Local Program } \\
\text { Reporting }\end{array}$ & 4 & 4 & 8 \\
\hline Production & Work Scope Procedures & 4 & 4 & 8 \\
\hline Production & Job Completion Verification & 4 & 1 & 5 \\
\hline Production & Subcontractors & 4 & 3 & 7 \\
\hline
\end{tabular}

The value of guidance in the form of a white paper was called into question by the expert panel as well as the value of webinars if they are too extensive in length. To that end, the guidance from this Strategy Guideline has been distilled into easy-to-read Fact Sheets in Appendix B through Appendix I, with links to additional information included. 


\section{Fact Sheets}

From the 15 highest rated Transition Points, eight were identified by IBACOS for expansion. Fact Sheets were created for these Transition Points in this Strategy Guideline. The use of the Fact Sheets to deliver information in small chunks was highly reccomended by the industry experts consulted. Fact Sheets are included as Appendix B through Appendix I of this Strategy Guideline, as follows:

- Training

- Equipment

- Customer Education

- Community Engagement

- On-Site Customer Interview

- Whole House Performance Assessment

- Customer Presentation Process

- Subcontractor and Vendor Relations

While not strictly technical in nature, Customer Education and Community Engagement were chosen as topics for the Fact Sheets based on their importance for early stages of the transition process. On-Site Customer Interview and Customer Presentation Process were chosen for their importance in developing a successful sales team.

The final four topics for the Fact Sheets-Whole House Performance Assessment, Equipment, Training, and Subcontractor and Vendor Relations - represent a solid base for the delivery of the whole house energy upgrade. 


\section{Conclusions and Next Steps}

HVAC companies need guidance on how to successfully transition from their traditional business strategy to one that includes WHPC. Although eight of the 15 highest-rated Transition Points are discussed in the appendices of this Strategy Guideline, contractors will need guidance in all aspects of their transition. The guidance offered in Building America reports is typically focused more on the technical aspects, with less emphasis on the business aspects. However, the two are not easily separated and must be considered together by the transitioning contractor as well as those developing further industry guidance.

Further development of the information contained in the Fact Sheets or the development of Fact Sheets for the remaining Transition Points would be the logical next steps to deliver the guidance needed. 


\section{References}

Bevilacqua Knight, Inc. (November 2003). 500-02-011, PIER Whole House Contracting Study. Oakland, CA: Bevilacqua Knight, Inc.

Contractor Excellence. Arlington, VA: Air Conditioning Contractors of America. www.contractorexcellence.com.

Home Energy. Berkeley, CA: Energy Auditor \& Retrofitter, Inc. www.homeenergy.org.

IBACOS, Inc. (2011). Final Expert Meeting Report: Transitioning Traditional HVAC Contractors to Whole House Performance Contractors, Building America Report. http://www.nrel.gov/docs/fy12osti/52665.pdf.

The Journal of Light Construction. Washington, D.C.: Hanley Wood LLC. http://www.jlconline.com. 


\section{Appendix A - Literature Search Results}

\section{Technical Training}

1) Energy Modeling Versus Reality Home Energy Magazine

ci. Mann, Steve. Energy Modeling Versus Reality, Home Energy Magazine, January/February 2010.

2) ENERGY STAR for Homes 3.0

Home Energy Magazine

ci. Mann, Steve. ENERGY STAR for Homes 3.0, Home Energy Magazine, January/February 2011.

3) Best Construction Details for Deep-Energy Retrofits Green Building Advisor

ci. Martin, Holladay. Best Construction Details for Deep-Energy Retrofits, Green Building Advisor.

http://www.greenbuildingadvisor.com/blogs/dept/musings/best-constructiondetails-deep-energy-retrofits, May 7, 2010.

4) How DoI...?

Home Energy Magazine

ci. Zimmerman, Allen. How Do I...? Home Energy Magazine, September/October 2007.

5) Keeping Educated in a Rapidly Changing Environment Home Energy Magazine

ci. Murphy, Casey. Keeping Educated in a Rapidly Changing Environment, Home Energy Magazine, September/October 2009.

6) Computer-Based Energy Analyst Training Home Energy Magazine

ci. Murphy, Casey. Keeping Educated in a Rapidly Changing Environment, Home Energy Magazine, September/October 2009.

7) From HVAC to Home Performance Contracting Home Energy Magazine

ci. Plympton, Patricia; Dagher, Leila. From HVAC to Home Performance Contracting, Home Energy Magazine, March/April 2007.

8) Guide to Training Programs for HPC (2005) Home Energy Magazine

ci. Home Energy. Guide to Training Programs for Home Performance Professionals, Home Energy Magazine, July/August 2005.

9) Guide to Training Programs for HPC (2009) Home Energy Magazine

ci. Home Energy. Guide to Training Programs for Home Performance Professionals, Home Energy Magazine, July/August 2009.

10) The 411 on Home Performance Contracting ACCA Contractor Excellence

ci. Contractor Excellence Magazine Staff. The 411 on Home Performance Contracting, ACCA Contractor Excellence.

http://www.contractorexcellence.com/326, September 1, 2009.

11) How to Properly Seal Ducts

Contracting Business

ci. Richardson, David. How to Properly Seal Ducts, Contracting Business. http://contractingbusiness.com/indoor_air_quality/content/, June 1, 2010. 
12) Quality Capacitors $=$ Quality Service

Contracting Business

ci. Parisi, Jim. Quality Capacitors = Quality Service, Contracting Business. http://contractingbusiness.com/service/content/quality-capacitors-0411/, April 1, 2011.

13) Saving Energy with Manual J and Manual D

Green Building Advisor

ci. Holladay, Martin. Saving Energy with Manual J and Manual D, Green Building Advisor. http://www.greenbuildingadvisor.com/blogs/dept/musings/savingenergy-manual-j-and-manual-d, August 13, 2010.

14) Sealing Ducts: What's Better, Tape or Mastic? Green Building Advisor

ci. Holladay, Martin. Sealing Ducts: What's Better, Tape or Mastic? Green Building Advisor. http://www.greenbuildingadvisor.com/blogs/dept/musings/sealingducts-what-s-better-tape-or-mastic, August 6, 2010.

15) When It's Time to Replace a Duct

Contracting Business

ci. Falke, Rob. When It's Time to Replace a Duct, Contracting Business. http://contractingbusiness.com/service/content/replacing_duct0918/, September 18, 2009.

16) 4 Pitfalls of Spray Foam Insulation Energy Vanguard

ci. Bailes, Allison. 4 Pitfalls of Spray Foam Insulation, Energy Vanguard. http://www.energyvanguard.com/blog-building-science-HERS-BPI/bid/25546/4Pitfalls-of-Spray-Foam-Insulation, June 29, 2010.

17) Air Sealing Tapes and Gaskets Green Building Advisor

ci. Holladay, Martin. Air Sealing Tapes and Gaskets, Green Building Advisor. http://www.greenbuildingadvisor.com/blogs/dept/musings/air-sealing-tapes-andgaskets, July 30, 2010.

18) Does It Make Sense to Move the Building Envelope... Energy Vanguard

ci. Bailes, Allison. Does It Make Sense to Move the Building Envelope to the Roofline? Energy Vanguard. http://www.energyvanguard.com/blog-buildingscience-HERS-BPI/bid/38272/Does-It-Make-Sense-to-Move-the-BuildingEnvelope-to-the-Roofline, May 23, 2011.

19) Creating a Conditioned Attic Green Building Advisor

ci. Holladay, Martin. Creating a Conditioned Attic, Green Building Advisor. http://www.greenbuildingadvisor.com/blogs/dept/musings/creating-conditionedattic, December 31, 2010.

20) A Do-It-Yourself Guide to Sealing and Insulating with... ENERGY STAR

ci. U.S. Environmental Protection Agency. A Do-It-Yourself Guide to Sealing and Insulating with ENERGY STAR, ENERGY STAR, August 2007.

21) Flash and Batt Builder Online

ci. Binsacca, Rich. Flash and Batt, Builder Online, http://www.builderonline.com/construction/flash-and-batt.aspx, February 10, 2011. 
22) How to Insulate a Low-Slope Roof

Green Building Advisor

ci. Gibson, Scott, How to Insulate a Low-Slope Roof, Green Building Advisor. http://www.greenbuildingadvisor.com/blogs/dept/green-building-blog/howinsulate-low-slope-roof, June 20, 2011.

23) Tighten Up Your Advice About Air Sealing Home Energy Magazine

ci. Newey, Mark. Tighten Up Your Advice About Air Sealing, Home Energy Magazine Special Issue 2005.

24) Comparing Open- and Closed-Cell Foams Home Energy Magazine

ci. Wyatt, Ed. Comparing Open- and Closed-Cell Foams, Home Energy Magazine, November/December 2010.

25) Homeowner's Guide to Insulating Owens Corning

ci. Owens Corning. Homeowner's Guide to Insulating, Pub. No. 10001528, September 2006. THE PINK PANTHER ${ }^{\mathrm{TM}}$.

26) What is Pressure?-Understanding Air Leakage Energy Vanguard

ci. Bailes, Allison. What Is Pressure?-Understanding Air Leakage, Energy Vanguard. http://www.energyvanguard.com/blog-building-science-HERSBPI/bid/35339/What-Is-Pressure-Understanding-Air-Leakage, January 24, 2011.

27) Are Dew-Point Calculations Really Necessary? Green Building Advisor

ci. Holladay, Martin. Are Dew-Point Calculations Really Necessary? Green Building Advisor. http:/www.greenbuildingadvisor.com/blogs/dept/musings/aredew-point-calculations-really-necessary, September 2010.

28) Controlling Humidity Green Building Advisor

ci. Wilson, Alex. Controlling Humidity, Green Building Advisor. http://www.greenbuildingadvisor.com/blogs/dept/energy-solutions/controllinghumidity, June 13, 2010.

29) Moisture Control Techniques for Wood-framed Homes Professional Builder

ci. Peavey, John. Moisture Control Techniques for Wood-framed Homes, Professional Builder, April 17, 2011.

30) Preventing Moisture Problems Green Building Advisor

ci. Wilson, Alex. Preventing Moisture Problems, Green Building Advisor. http://www.greenbuildingadvisor.com/blogs/dept/energy-solutions/preventingmoisture-problems, September 30, 2008.

31) Seal \& Supply Solves Bathroom Mold Strife Contracting Business

ci. O’Neil, Kevin. Seal \& Supply Solves Bathroom Mold Strife, Contracting Business. http://contractingbusiness.com/indoor_air_quality/content/seal-supplybathroom-mold-0411/. April 1, 2011. 
ci. Holladay, Martin. Radiant Barriers: A Solution in Search of a Problem, Green Building Advisor. http://www.greenbuildingadvisor.com/blogs/dept/musings/radiant-barrierssolution-search-problem, September 24, 2010.

33) Crawl Space Building Science Energy Vanguard

ci. Bailes, Allison. Crawl Space Building Science, Energy Vanguard. http://www.energyvanguard.com/crawl-space-building-science/, 2011.

34) Designing a Good Ventilation System Green Building Advisor

ci. Holladay, Martin. Designing a Good Ventilation System, Green Building Advisor. http://www.greenbuildingadvisor.com/blogs/dept/musings/designinggood-ventilation-system, June 15, 2010.

35) Don't Let Your Attic Suck-Power Attic Ventilators... Energy Vanguard

ci. Bailes, Allison. Don't Let Your Attic Suck-Power Attic Ventilators Are a Bad Idea, Energy Vanguard Blog. http://www.energyvanguard.com/blog-buildingscience-HERS-BPI/bid/38676/Don-t-Let-Your-Attic-Suck-Power-AtticVentilators-Are-a-BadIdea? source=Blog Email \%5bDon $\%$ e2\%80\%99t+Let+Your+Attic\%5d, June 08, 2011.

36) Mechanical Ventilation: Breathe Energy Circle

ci. Energy Circle Staff. Mechanical Ventilation: Breathe, Energy Circle. http://www.energycircle.com/learn/air-quality/mechanical-ventilation, August 18, 2009.

37) Reexamining Roof Ventilation Home Energy Magazine

ci. Woods, Tony. Reexamining Roof Ventilation, Home Energy Magazine, November/December 2000.

38) Nailing Window Flanges Through Foam Green Building Advisor

ci. Holladay, Martin. Nailing Window Flanges Through Foam, Green Building Advisor. http://www.greenbuildingadvisor.com/blogs/dept/musings/nailingwindow-flanges-through-foam, May 20, 2011.

39) 'Superwindows' To the Rescue? Green Building Advisor

ci. Roberts, Tristan. 'Superwindows' To the Rescue? Green Building Advisor. http://www.greenbuildingadvisor.com/blogs/dept/energysolutions/superwindows-rescue, June 7, 2011. 
40) Understanding What Makes High-Performance Windows...

Green Building Advisor

ci. Roberts, Tristan. Understanding What Makes High-Performance Windows Perform, Green Building Advisor.

http://www.greenbuildingadvisor.com/blogs/dept/energy-

solutions/understanding-what-makes-high-performance-windows-perform,

May 23, 2011.

41) Using Window Stickers to Get What's Right for You

Green Building Advisor

ci. Roberts, Tristan. Using Window Stickers to Get What's Right for Your Climate, Green Building Advisor.

http://www.greenbuildingadvisor.com/blogs/dept/energy-solutions/usingwindow-stickers-get-whats-right-your-climate, May 31, 2011.

\section{Equipment Requirements}

1) Products List ACCA Contractor Excellence

ci. Contractor Excellence Magazine Staff. Products List, ACCA Contractor Excellence. http://www.contractorexcellence.com/category/sections/products, last updated April 5, 2011.

2) Minneapolis Blower Door The Energy Conservatory

ci. Brochure: Minneapolis Blower Door, The Energy Conservatory, 2010.

3) testo $317-3$ Testo Inc.

ci. Brochure: testo 317-3, Testo, Inc., 35 Ironia Rd., Flanders, NJ 07836.

4) Leakator 10 Combustible Gas Leak Detector Bacharach

ci. Brochure: Bacharach, Leakator 10 Combustible Gas Leak Detector, August 2002. www.bacharach-inc.com.

5) CD200 Combustible Gas Leak Detector UEi

ci. Brochure: UEi, CD200 Combustible Gas Leak Detector, UEi 2007. www.ueitest.com.

6) Combustible-Gas Leak Detectors Home Energy Magazine

ci. Raymer, Paul. Combustible-Gas Leak Detectors, Home Energy Magazine, May/June 2011.

7) Minneapolis Duct Blaster The Energy Conservatory

ci. Brochure: Minneapolis Duct Blaster, The Energy Conservatory, 2010.

8) REM/Rate Architectural Energy

ci. Architectural Energy. REM/Rate, Architectural Energy Corporation. http://www.archenergy.com/products/remrate, 2010.

9) Right-J Wright Soft

ci. Wright Soft. Right-J. http://www.wrightsoft.com/products/right-j.aspx 2011. 
10) Fluke TiS Building Diagnostic Thermal Imaging Scanner

Fluke

ci. Brochure: Fluke TiS Building Diagnostic Thermal Imaging Scanner, (C2010

Fluke Corporation. Printed in U.S.A., 6/2010, 3804542A D-EN-N.

11) IR-Worth a Thousand Words

Home Energy Magazine

ci. Collins, Maureen. IR-Worth a Thousand Words, Home Energy Magazine, September/October 2008.

12) Dispatching Software: The Wind Beneath Your Wheels

Contracting Business

ci. McIver, Terry. Dispatching Software: The Wind Beneath Your Wheels,

Contracting Business. http://contractingbusiness.com/service/dispatchingsoftware-0411/index.html, April 1, 2010.

13) The Right Tools For the Job ACCA Contractor Excellence

ci. Contractor Excellence Magazine Staff. The Right Tools for the Job, ACCA Contractor Excellence. http://www.contractorexcellence.com/4595, March 31, 2011.

14) Bacharach Equipment Manuals Bacharach

15) MN Equipment Manuals The Energy Conservatory

16) Retrotec Equipment Manuals Retrotec

17) Combustible-Gas Leak Detectors Home Energy Magazine

ci. Raymer, Paul. Combustible-Gas Leak Detectors, Home Energy Magazine, May/June 2011.

\section{Vendor Relations}

1) A Way to Build Great and Lasting Vendor Relationships $\quad$ R.B. Lewis \& Associates

ci. Lewis, Ron. A Way to Build Great and Lasting Vendor Relationships-Vendors Measuring Your Performance, R.B. Lewis \& Associates.

www.rblewisassociates.com, 12/2010.

\section{Strategic Planning}

1) Building a Multifamily Home Performance Program Home Energy Magazine

ci. Summerford, Julieann. Building a Multifamily Home Performance Program, Home Energy Magazine, May/June 2011.

2) The Business Case for 'Smaller is Better' Green Building Advisor

ci. Strong, Michael. The Business Case for 'Smaller Is Better,' Green Building Advisor. http://www.greenbuildingadvisor.com/blogs/dept/businessadvisor/business-case-smaller-better, December 20, 2010. 
3) Contractor Takes Holistic Approach

ACCA Contractor Excellence

ci. Contractor Excellence Magazine Staff. Contractor Takes Holistic Approach, ACCA Contractor Excellence. http://www.contractorexcellence.com/2817, September 20, 2010.

4) Remodeling a Business Home Energy Magazine

ci. James, Mary. Remodeling a Business, Home Energy Magazine, September/October 2004.

5) Learning and Working Our Way to Success Home Energy Magazine

ci. Rogers, Mike. Learning and Working Our Way to Success, Home Energy Magazine Special Issue 2005.

6) My $8^{\text {th }}$ Commandment: Never Let the Client... Green Building Advisor

ci. Strong, Michael. My $8^{\text {th }}$ Commandment: Never Let the Client.... Green Building Advisor. http://www.greenbuildingadvisor.com/blogs/dept/business-advisor/my8th-commandment-never-let-client, October 11, 2010.

7) Practical Lessons from a Home Performance Contract Home Energy Magazine

ci. Knight, Bob; Locke, Time. Practical Lessons from a Home Performance Contractors Association, Home Energy Magazine.

8) The Making of a Small Business Owner Home Energy Magazine

ci. Lupberger, David. The Making of a Small Business Owner, Home Energy Magazine HPC-A Business Development Guide.

9) So You Want to Be a Home Performance Contractor Home Energy Magazine

ci. Thomas, Greg. So You Want to Be a Home Performance Contractor, Home Energy Magazine HPC-A Business Development Guide.

10) Guaranteed to Succeed Home Energy Magazine

ci. James, Mary. Guaranteed to Succeed, Home Energy Magazine, March/April 2005.

11) A Whirlwind Startup Home Energy Magazine

ci. James, Mary. A Whirlwind Startup, Home Energy Magazine, November/December 2004.

12) Greening of a Home Performance Contractor Home Energy Magazine

ci. Thomas, Greg. Greening of a Home Performance Contractor, Home Energy Magazine, May/June 2005.

13) Internet Marketing ACCA Contractor Excellence

ci. Hudson, Adams. Internet Marketing. ACCA Contractor Excellence. http://www.contractorexcellence.com/5254, May 9, 2011.

14) Marketing Essentials Home Energy Magazine

ci. Home Energy. Marketing Essentials, Home Energy Magazine HPC-A Business Development Guide. 
15) Help Customers Choose High Efficiency

ACCA Contractor Excellence

ci. Turpin, Joanna. Help Customers Choose High Efficiency, ACCA Contractor Excellence. http://www.contractorexcellence.com/1940, July 6, 2010.

16) How to Sell Comfort in a Green World C ontracting Business

ci. Givens, Alan. How to Sell Comfort in a Green World, Contracting Business. http://contractingbusiness.com/indoor_air quality/content/sell-comfort-green0609/index.html, June 1, 2009.

17) How to Win the Job Home Energy Magazine

ci. Gorman, Mike. How to Win the Job, Home Energy Magazine, September/October 2009.

18) Sales Strategies Home Energy Magazine

ci. Kornbluth, Dick; Taylor, Larry; Hughes, Darin; Seville, Carl. Sales Strategies, Home Energy Magazine HPC-A Business Development Guide.

19) Selling High-Performance Audits Home Energy Magazine

ci. Cooke, Gord. Selling High-Performance Audits, Home Energy Magazine, September/October 2009.

20) The Five Ingredients in a Sale ACCA Contractor Excellence

ci. Rees, Michael. The Five Ingredients in a Sale, ACCA Contractor Excellence. http://www.contractorexcellence.com/5142, April 25, 2011.

21) Tips for Selling Green ACCA Contractor Excellence

ci. Contractor Excellence Magazine Staff. Tips for Selling Green, ACCA Contractor Excellence. http://www.contractorexcellence.com/3911, January 3, 2011.

22) Two Ears, One Mouth: Listen Your Way to Increased... Contracting Business

ci. Greer, Charlie. Two Ears, One Mouth: Listen Your Way to Increased Sales, Contracting Business. http://contractingbusiness.com/columnists/greer/two-earsone-mouth-0411/, April 1, 2011.

\section{Customer Education Process}

1) Use Your Customers as Part of Your Sales Force Building Performance Institute

ci. Building Performance Institute. Use Your Customers as Part of Your Sales Force. http://www.bpi.org/news_pm july_09.aspx, Building Performance Institute 2010.

2) Winter Relief Assistance Program APPRISE

ci. APPRISE. Winter Relief Assistance Program. http://www.appriseinc.org/reports/Final\%20PPL\%20WRAP\%20Evaluation\%20 Report.pdf, APPRISE, April 2006.

3) What Every Consumer Really Wants to Know Contracting Business

ci. Falke, Rob. What Every Consumer Really Wants to Know, Contracting Business, February 3, 2010. 
4) Maine Home Performance Technical Standards

ENERGY STAR

ci. Boothby, Tom; Karg, Rick. Maine Home Performance Technical Standards. http://www.karg.com/pdf/MHP\%20Standards\%20Book\%20Final\%200908\%20v 2.pdf, Maine Home Performance with ENERGY STAR Standards, September 2008, v2.

5) Energy Efficient Mortgages

Home Energy Magazine

ci. Mann, Steve. Energy Efficient Mortgages, Home Energy Magazine, March/April 2009.

6) Best-Kept Secret in the Mortgage Industry

Home Energy Magazine

ci. Baden, Steve. Best-Kept Secret in the Mortgage Industry, Home Energy Magazine, January/February 2007.

7) A Wisconsin Usonian Home: 37 Years of Energy History Home Energy Magazine

ci. Foell, Wesley K. A Wisconsin Usonian Home: 37 Years of Energy History, Home Energy Magazine, July/August 2009.

\section{Community Engagement}

1) Community Energy Efficiency Program Best Practices...

Clean Energy Solution

ci. Clean Energy Solution. Community Energy Efficiency Program Best Practices Marketing Strategies. http://www.cleanenergysol.com/news/news_confrences.html, September 2010.

2) Inform Homeowner Participation in California Energy... Action Research

ci. Action Research. Inform Homeowner Participation in California EnergyEfficiency Home Improvement Programs. http://www.takeactionresearch.com, $7 / 26 / 2010$.

3) The DC Project WeatherizeDC

ci. WeatherizeDC. The DC Project. http://www.WeatherizeDC.org, 2010 Annual Report.

\section{Assessment Arrival Process}

1) Combustion Appliance Testing: Why, How, When? Home Energy Magazine

ci. Sterner, A. Tamasin. Combustion Appliance Testing: Why, How, When? Home Energy Magazine, November/December 2010.

2) Combustible-Gas Leak Detectors Home Energy Magazine

ci. Raymer, Paul. Combustible-Gas Leak Detectors, Home Energy Magazine, May/June 2011.

3) Letters Home Energy Magazine

ci. Sterner, Tamasin; Stratton, Chris. Letters, Home Energy Magazine, May/June 2011. 


\section{On-Site Customer Interview}

1) Be a Detective

Contracting Business

ci. Coop, Tina. Be a Detective, and 'Investigate' During Service Calls, Contracting Business. http://contractingbusiness.com/service/content/be-detectiveinvestigate-0311/, March 1, 2011.

2) IAQ for Homes Contracting Business

ci. Esch, Bill. IAQ for Homes, Contracting Business. http://contractingbusiness.com/indoor air_quality/content/iaq-homes-0909/, September 1, 2009.

\section{Whole House Assessment}

1) Kick the Can! No Recessed Lights in the Building Envelope Energy Vanguard

ci. Bailes, Allison. Kick the Can! No Recessed Lights in the Building Envelope, Energy Vanguard. http://www.energyvanguard.com/blog-building-scienceHERS-BPI/bid/37246/Kick-the-Can-No-Recessed-Lights-in-the-BuildingEnvelope, April 4, 2005.

2) Multifamily Assessment Home Energy Magazine

ci. Genge, Colin. Measuring Leakage in Multifamily Buildings, Home Energy Magazine, September/October 2007.

3) Sealed Attics? Home Energy Magazine

ci. Coble, Brian. Sealed Attics? Home Energy Magazine, January/February 2010.

4) The Telltale Sign of Infiltration Energy Vanguard

ci. Bailes, Allison. The Telltale Sign of Infiltration, Energy Vanguard. http://www.energyvanguard.com/blog-building-science-HERSBPI/bid/30707/The-Telltale-Sign-of-Infiltration, September 20, 2010.

5) Plan Your Attack Energy Circle

ci. Energy Circle Staff. Plan Your Attack, Energy Circle. http://www.energycircle.com/learn/air-sealing/plan-your-attack, August 18, 2009.

6) An Easier Way to Measure Duct Leaks Home Energy Magazine

ci. Sherman, Max; Walker, Iain. An Easier Way to Measure Duct Leaks, Home Energy Magazine, September/October 2002.

7) Grupe Homes Enters the Whole-House Retrofit Market Home Energy Magazine

ci. Gilbride, Therease; Hefty, Marye. Grupe Homes Enters the Whole-House Retrofit Market, Home Energy Magazine, March/April 2010.

8) Baseline Standard Practices Survey: Task Report Team IBTS

ci. Team IBTS. Baseline Standard Practices Survey: Task Report. www.huduser.org, January 30, 2006. 
9) New Construction Report Card II

Home Energy Magazine

ci. Chitwood, Rick; Hoeschele Marc. New Construction Report Card, Part II, Home Energy Magazine, March/April 2003.

10) Testing Duct Leakage Home Energy Magazine

ci. Francisco, Paul; Pigg, Scott. Testing Duct Leakage, Home Energy Magazine, March/April 2009.

11) Formicary Corrosion-A Primer ACCA

ci. Prather, Donald. Formicary Corrosion - A Primer, Air Conditioning Contractors of America, 2009.

12) Passive Solar Heating Green Building Advisor

ci. Wilson, Alex. Passive Solar Heating, Green Building Advisor.

http://www.greenbuildingadvisor.com/blogs/dept/energy-solutions/passive-solarheating, November 4, 2008.

13) Temperature Differentials in Residential Applications

ACCA

ci. Prather, Donald. Temperature Differentials in Residential Applications, Air Conditioning Contractors of America, 2009.

14) Why You Need HVAC Load Calculations Energy Vanguard

ci. Bailes, Allison. Why You Need HVAC Load Calculations, Energy Vanguard. http://www.energyvanguard.com/hvac-design/, 2011.

15) An Introduction to Indoor Air Quality EPA

ci. U.S. Environmental Protection Agency. An Introduction to Indoor Air Quality,

U.S. Environmental Protection Agency. http://www.epa.gov/iaq/voc.html, March 22, 2011.

16) Roof Topography \& Ice Damming Home Energy Magazine

ci. Labbé, Greg. Roof Topography \& Ice Damming, Home Energy Magazine, January/February 2010.

17) Indoor Environmental Quality Daydreams and Night... Home Energy Magazine

ci. Shaw, Kirsten. Indoor Environmental Quality Daydreams and Nightmares, Home Energy Magazine, May/June 2011.

18) Attic Stairs-A Mind-Blowing Hole in Your Building... Energy Vanguard

ci. Bailes, Allison. Attic Stairs-A Mind-Blowing Hole in Your Building Envelope, Energy Vanguard. http://www.energyvanguard.com/blog-building-scienceHERS-BPI/bid/34932/Attic-Stairs-A-Mind-Blowing-Hole-in-Your-BuildingEnvelope, January 7, 2011. 
19) Does It Make Sense to Move the Building Envelope to...

Energy Vanguard

ci. Bailes, Allison. Does It Make Sense to Move the Building Envelope to the Roofline? Energy Vanguard Blog. http://www.energyvanguard.com/blogbuilding-science-HERS-BPI/bid/38272/Does-It-Make-Sense-to-Move-theBuilding-Envelope-to-the-

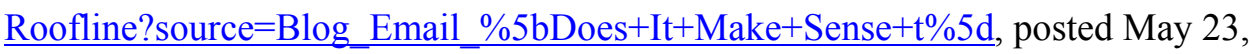
2011.

20) Cold Interior Walls, Useless Insulation, and Building Science Energy Vanguard

ci. Bailes, Allison. Cold Interior Walls, Useless Insulation, and Building Science, Energy Vanguard. http://www.energyvanguard.com/blog-building-scienceHERS-BPI/bid/33564/Cold-Interior-Walls-Useless-Insulation-and-BuildingScience, December 3, 2010.

21) Flat or Lumpy —How Would You Like Your Insulation? Energy Vanguard

ci. Bailes, Allison. Flat or Lumpy — How Would You Like Your Insulation? Energy Vanguard. http://www.energyvanguard.com/blog-building-science-HERSBPI/bid/25547/Flat-or-Lumpy-How-Would-You-Like-Your-Insulation, June 28, 2010.

22) Infrared Thermography: (Nearly) A Daily Tool Home Energy Magazine

ci. Snell, John. Infrared Thermography: (Nearly) A Daily Tool, Home Energy Magazine, March/April 2008.

23) RESNET \& Infrared Thermography Home Energy Magazine

ci. Snell, John. RESNET \& Infrared Thermography, Home Energy Magazine, January/February 2010.

24) Successful Warm-Weather Infrared Inspections Home Energy Magazine

ci. Schwoegler, Matt. Successful Warm-Weather Infrared Inspections, Home Energy Magazine, May/June 2011.

25) Check Your Showerhead! Home Energy Magazine

ci. Hain, Lexie; Harrod Jon. Check Your Showerhead! Home Energy Magazine, November/December 2007.

\section{Customer Presentation Package}

1) Energy Monitoring; Product Review Energy Circle

ci. Energy Circle Staff; Harrison, Tom. Energy Monitoring; Product Review, Energy Circle. http://www.energycircle.com/learn/energy-monitoring, Energy Monitoring: December 18, 2009; Product Reviews: September 13, 2010.

2) How to Sell Green Upgrades Green Building Advisor

ci. Strong, Michael. How to Sell Green Upgrades..., Green Building Advisor. http://www.greenbuildingadvisor.com/blogs/dept/business-advisor/how-sellgreen-upgrades-radiant-barrier-paints, January 25, February 8, March 9, June 9, 2011. 
3) Making Your Home Safer with a Sealed Combustion...

Energy Vanguard

ci. Bailes, Allison. Making Your Home Safer with a Sealed Combustion Closet, Energy Vanguard. http://www.energyvanguard.com/blog-building-scienceHERS-BPI/bid/35938/Making-Your-Home-Safer-with-a-Sealed-CombustionCloset, February 11, 2011.

4) The Ductless Mini-Split Heat Pump's Big Brother Energy Vanguard

ci. Laumer-Giddens Chris. The Ductless Mini-Split Heat Pump's Big Brother-CITY MULTI, Energy Vanguard. http://www.energyvanguard.com/blog-buildingscience-HERS-BPI/bid/38008/The-Ductless-Mini-Split-Heat-Pump-s-BigBrother-CITY-MULTI, May 11, 2011.

5) Retrofit Your Existing Building with High Performance... Quad-Lock Building Systems

ci. Quad-Lock Building Systems. Retrofit Your Existing Building with High Performance Insulation, Quad-Lock Building Systems version 1.4, 01/2010.

6) 10 Things You Should Put in Your House Builder Online

ci. Maynard, Nigel. 10 Things You Should Put in Your House in 2010, Builder Online. http://www.builderonline.com/products/10-things-you-should-put-inyour-house-in-2010.aspx, December 1, 2009.

7) Products Guide ACCA Contractor Excellence

ci. Contractor Excellence Magazine Staff. Products Guide, ACCA Contractor Excellence. http://www.contractorexcellence.com/category/sections/products, last updated April 5, 2011.

8) Residential: Ducted/Non-ducted Air Conditioning, Geo... Contracting Business

ci. Kelly, Kate. Residential: Ducted/Non-ducted Air Conditioning, Geothermal Systems; Commercial: Chillers, VRF Systems, and Applied Equipment, Contracting Business.

http://contractingbusiness.com/residential/content/residential-ducted-nonductedair-conditioning-0310/index.html, March 1, 2010.

9) 2009/2010 Guide to High Efficiency Gas Furnaces Contracting Business

ci. Contracting Business.com. 2009/2010 Guide to High Efficiency Gas Furnaces. http://contractingbusiness.com/images/0909HeatingGuide.pdf, September, 2009.

10) 2011 Guide to High Efficiency Air Conditioning Contracting Business

ci. Contracting Business.com. 2011 Guide to High Efficiency Air Conditioning. http://contractingbusiness.com/CB0411-2011-AC-guide.pdf, April 4, 2011.

11) New Green Building Products Green Building Advisor

ci. Holladay, Martin. New Green Building Products, Green Building Advisor. http://www.greenbuildingadvisor.com/blogs/dept/musings/new-green-buildingproducts, September 10, 2010. 
12) Martin's Useless Products List

Green Building Advisor

ci. Holladay, Martin. Martin's Useless Products List, Green Building Advisor. http://www.greenbuildingadvisor.com/blogs/dept/musings/martin-s-uselessproducts-list, September 25, 2010.

13) A Roundup of New Green Building Products Green Building Advisor

ci. Holladay, Martin. A Roundup of New Green Building Products, Green Building Advisor. http://www.greenbuildingadvisor.com/blogs/dept/musings/roundupnew-green-building-products, March 25, 2010.

14) Energy Savers: Thermostats and Control Systems U.S. DOE

ci. U. S. Department of Energy. Energy Savers: Thermostats and Control Systems, U.S. Department of Energy.

http://www.energysavers.gov/your home/space heating cooling/index.cfm/myto $\mathrm{pic}=12720,02 / 09 / 2011$.

15) Using Thermostats

Green Building Advisor

ci. Wilson, Alex. Using Thermostats, Building Green Advisor. http://www.greenbuildingadvisor.com/blogs/dept/energy-solutions/usingthermostats, March 24, 2009.

16) Reduce Hot Water Use for Energy Savings U.S. DOE

ci. Energy Savers. Reduce Hot Water Use for Energy Savings. http://www.energysavers.gov/your_home/water_heating/index.cfm/mytopic $=130$ 50, U.S. Department of Energy, 2/9/2011.

17) Tankless Water Heaters Getting More Efficient Professional Builder

ci. Corey, Jason. Tankless Water Heaters Getting More Efficient, Professional Builder, May 31, 2011.

18) Affordable Window Retrofits Green Building Advisor

ci. Yost, Peter. Affordable Window Retrofits, Green Building Advisor. http://www.greenbuildingadvisor.com/blogs/dept/green-communities/affordablewindow-retrofits, June 21, 2011.

19) The Pros and Cons of Window Films Builder Online

ci. Royer, Evelyn. The Pros and Cons of Window Films, Builder Online. http://www.builderonline.com/green-products/the-pros-and-cons-of-windowfilms.aspx, May 4, 2011.

\section{Assessment Report}

1) Converting Between CFM 50 and Natural Airflow Building Performance Institute

ci. Building Performance Institute. Converting Between CFM 50 and Natural Airflow, Building Performance Institute, 4/07.

2) Distribution Efficiency Look-Up Table Building Performance Institute

ci. Building Performance Institute. Distribution Efficiency Look-Up Table, Building Performance Institute, 4/07. 
3) Ensuring ASHRAE 62.2-2010 Ventilation Compliance... ACCA

ci. Prather, Donald. Ensuring ASHRAE 62.2-2010 Ventilation Compliance for Residential Structures, Air Conditioning Contractors of America, 2010.

4) Sick Building Syndrome Building Performance Institute

ci. Building Performance Institute. Sick Building Syndrome, U.S. Environmental Protection Agency, February 1991.

5) R-Value Calculations Building Performance Institute

ci. Building Performance Institute. R-Value Calculations, Building Performance Institute, 4/07.

\section{State or Local Program Reporting}

1) Home Performance with ENERGY STAR Customer Incent

ENERGY STAR

ci. Consumers Energy. Home Performance with ENERGY STAR Customer Incentives, ENERGY STAR, 1/12/11.

2) Want Energy-Saving Improvements in Your Home?... San Francisco Chronicle

ci. Hibble, Anna Marie. Want Energy-Saving Improvements In Your Home? The Feds May Help..., San Francisco Chronicle, 4/21/2011.

3) Clean Up Your Ecological Footprint Home Energy Magazine

ci. Gunshinan, Jim. Clean Up Your Ecological Footprint, Home Energy Magazine, May/June 2005.

4) Incentives and Rebates for Energy Efficient Windows... Efficient Windows Collaborative

ci. Efficient Windows Collaborative. http://www.efficientwindows.org, January 2011.

\section{Work Scope/Procedures}

1) Combustion Safety Test Building Performance Institute

ci. Saturn Resource Management, BPI Combustion Test Instructions, SRMI. http://www.srmi.biz.

2) Combustion Safety Test (2) Building Performance Institute

ci. Building Performance Institute. Combustion Safety Test Procedure for Vented Appliances, Building Performance Institute, 4/07.

3) Guide to Performance Test Building Performance Institute

ci. Building Performance Institute. Building Analyst-Guide to Performance Test, Building Performance Institute, 1/26/07.

4) Sick Building Syndrome EPA

ci. U.S. Environmental Protection Agency. Sick Building Syndrome, Environmental Protection Agency—Research and Development, February 1991. 


\section{Job Completion Verification}

1) Improving Your Home Performance Contracting Bus...

Home Energy Magazine

ci. Abrey, David. Improving Your Home Performance Contracting Business Through Quality Management, Home Energy Magazine, September/October 2009.

2) Baseline Standard Practices Survey: Task Report Team IBTS

ci. Team IBTS. Baseline Standard Practices Survey: Task Report. www.huduser.org, January 30, 2006.

3) RESNET Quality Assurance Home Energy Magazine

ci. Gunshinan, Jim. RESNET Quality Assurance, Home Energy Magazine, July/August 2005. 
\begin{tabular}{l|l} 
U.s. Department of & Energy Efficiency \& \\
Renewable Energy
\end{tabular}

Appendix B - Training 


\section{Operational Area: Business Planning/Process}

\section{Work Activity: Training}

\section{Issue}

Transitioning into home performance contracting requires training in the basics of building science, as well as a comprehensive understanding of the various aspects of home performance. Home performance contractors should be able to identify problems not just with a single residential system but anywhere in the home using a "house as a system" approach. Certified training providers can teach these concepts through courses that present building performance standards such as the Building Performance Institute (BPI)'s Building Analyst standards and RESNET's Home Energy Rating System (HERS) Rater standards. Conferences also can be an excellent source of training.

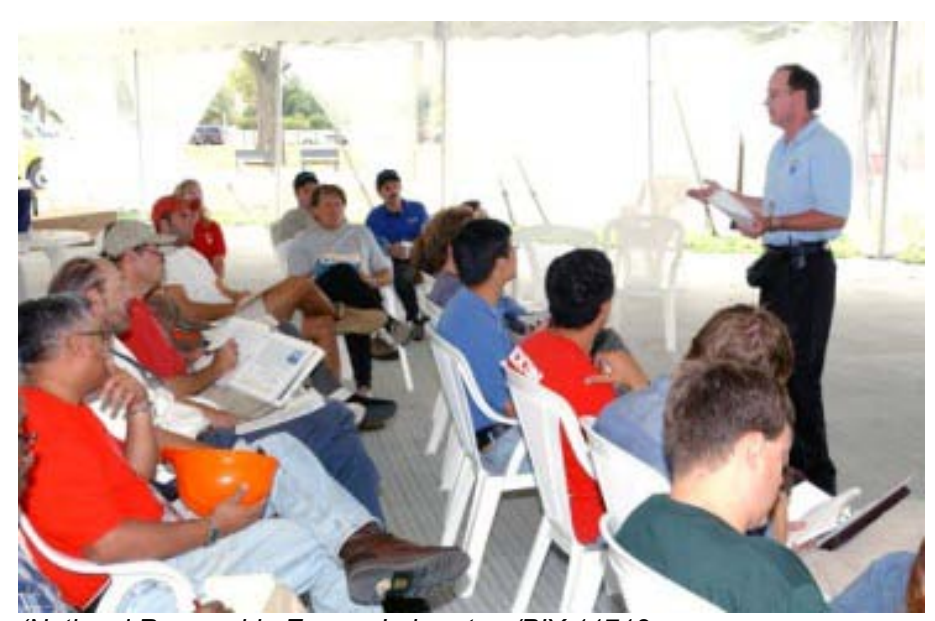

(National Renewable Energy Laboratory/PIX 11712

http://www.nrel.gov/data/pix/Jpegs/11712.jpg)

\section{Building Science}

A strong and comprehensive home performance training course should be built on a foundation of building science concepts. Key themes should include principles of energy conservation and efficiency; building shell construction and diagnostics; insulation and heat transfer; air pressure and airflow; humidity and moisture; HVAC and water heating essentials; lighting and appliances; and occupant health and safety. Thoroughly understanding how these principles operate and interact will help you evaluate the myriad of situations you may come across in actual homes and will allow you to devise the best possible solutions on a case-by-case basis.

\section{Topics Covered}

Beyond the basics, training in home performance should cover a wide range of specific topics. A good course will focus on not only energy efficiency and building science but also health, safety, and comfort. A full curriculum will generally include all of the following topic areas:

- Heat transfer and heat flow: types of heat flow (conduction, convection, and radiation); energy transformation into heat; heat loss.

- Air pressure and air flow: air pressure evaluation; leakage diagnostics; air sealing; duct leakage; blower door testing.

- $\quad$ Building envelope: thermal boundary; insulation; solar gain; external air infiltration.

- Moisture management: infiltration of humid air; internally generated moisture; rainwater infiltration; vapor diffusion.

- Indoor air quality: indoor pollutants; pollutant distribution; thermal comfort; ventilation.

Learning these concepts fully also requires some handson training in the field, which in some cases may be combined with classroom instruction, including the following:

- Blower door setup and operation

- Use of a smoke pencil to detect air leaks

- Analyzing pressure differentials with a digital manometer

- Diagnosing duct leakage with a duct blaster

- Assessing air distribution balance with a flow hood

- Installing and using carbon monoxide detectors and gas sniffers

- Conducting a thorough combustion test

Beyond training in the fundamental concepts listed above, a successful home performance contractor should become educated in areas such as building codes and regulations, as well as available incentive programs. To be competitive, the contractor should stay on top of best practices from a business perspective as well. 


\section{Certifications}

There are many different certifications available, depending on your needs, clients, market, and programs in which you may want to work. These range from professional certifications within a particular slice of the energy efficiency industry (e.g., renewables, building operations, commissioning, auditing) to certifications in associated industries, such as green building. The two building performance certifications most recognized by state and federal programs today are those issued by BPI and RESNET (the Residential Energy Service Network). Historically, BPI focused primarily on existing buildings, while RESNET's HERS certification focused more on new construction. However, more recently that line has been blurred.

There are still some differences between the two programs, although they are more subtle. Both certifications now include a combustion safety requirement to identify unsafe space heating and water heating systems. BPI also includes identifying natural gas leaks and monitoring ambient carbon monoxide, while HERS Rater training emphasizes duct testing with the use of a duct blaster. In addition, HERS Raters are trained to use software that will provide a house with an overall efficiency rating. RESNET operates a quality assurance system for all HERS Raters.

Nonetheless, because the fundamental concepts in both types of training are mostly the same, it is worth examining the curriculum of whichever course you choose to make sure it includes the items you wish to learn. One reason to choose a particular certification may also be that it is required by a particular incentive program that you can tap into when marketing your services to potential customers.

Training and preparing for a professional certification usually takes at least a week of time at a cost of about $\$ 1,500$, not including time away from your business. Qualifying for the certification is another $\$ 1,000$ or so.

Note that both standards programs also carry continuing education requirements. HERS Raters must complete 12 hours of classroom instruction every year, while BPI Building Analysts must recertify every three years with 10 to 30 hours of classroom instruction and an option for written examination.

\section{Conferences}

Local, regional, and national conferences are an excellent training resource. While some provide pre- or posttraining toward certification, most are useful primarily for continuing education, networking with others in the field, and keeping abreast of the latest developments in the building performance world. Some of the more popular building science conferences include the following:

- ACEEE Hot Water Forum

- ACEEE Summer Study in Buildings

- Affordable Comfort Conference

- Better Buildings By Design (EVT)

- Building Energy (NESEA)

- Energy and Environmental Building Assoc.

- GreenBuild (USGBC)

- National Green Building Conference (NAHB)

- RESNET Conference

\section{Article Snippets}

Home Energy Magazine:

Keeping Educated in a Rapidly Changing Environment

"Home performance contracting addresses a multitude of issues that affect homeowners, including their home's energy use, moisture movement, indoor air quality (IAQ), comfort, and durability. It is not sufficient for a contractor to be a jack-of-all-building-trades. He or she must be a master of most, and must know where to look when searching for answers to difficult questions." 9/4/2009 http://www.homeenergy.org/show/article/nav/homeperformance/id/657

\section{From HVAC to Home Performance Contracting}

Matthew Holtkamp, founder of Holtkamp Heating and Air Conditioning (HHVAC) in Atlanta, Georgia, obtained home performance training to serve his customers better.

"With the new testing equipment and procedures, Holtkamp could pin down the source of any HVAC problem and find a solution based on the condition of the whole house. Before the training, for example, if the client complained of high energy bills and dust, he would install a new high-efficiency furnace and recommend a new furnace filter for dust. After the training, he and his employees would diagnose the problem as duct leakage using a blower door assessment, and seal the ducts before installing the new furnace."10/3/2007 http://www.homeenergy.org/show/article/nav//id/373 


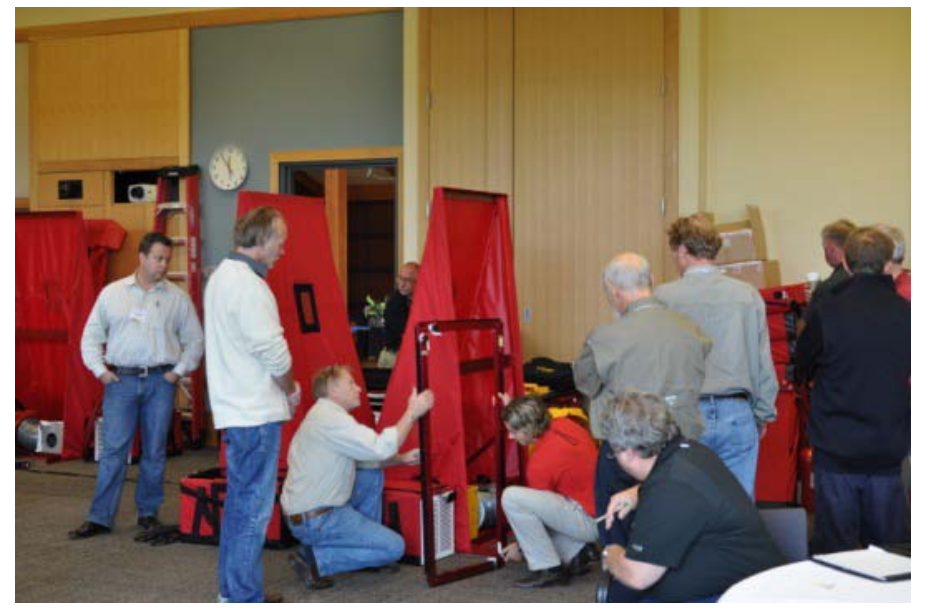

(Lawrence Berkeley National Laboratory, http://homeenergypros. lbl.gov/photo/q32-ductester-system/prev?context=user)

\section{Resources}

BPI Training:

http://www.bpi.org/schedules training.aspx

RESNET HERS Rater Training: http://www.resnet.us/programs/training

"House Simulator Tent."

http://homeenergypros.lbl.gov/photo/q32-ductestersystem/prev?context=user 
\begin{tabular}{l|l} 
u.s. department of & $\begin{array}{l}\text { Energy Efficiency \& } \\
\text { Renewable Energy }\end{array}$
\end{tabular}

\section{Appendix C - Equipment}




\section{HVAC Transition Guide for Building Performance Contractors}

\section{Operational Area: Business Planning/Process}

\section{Work Activity: Equipment}

\section{Issue}

Transitioning into home performance contracting will require some investment in the tools necessary to diagnose and complete a comprehensive home performance job. The advantage of acquiring and learning how to use these devices is that you will be much better equipped to identify any issues in a customer's home and then upgrade them effectively and safely. From air leakage to excess moisture to health and safety problems, having the right tools in your kit will enable you to conduct a thorough assessment, avoid missing any issues, and repair the home's deficiencies.

\section{Blower Door}

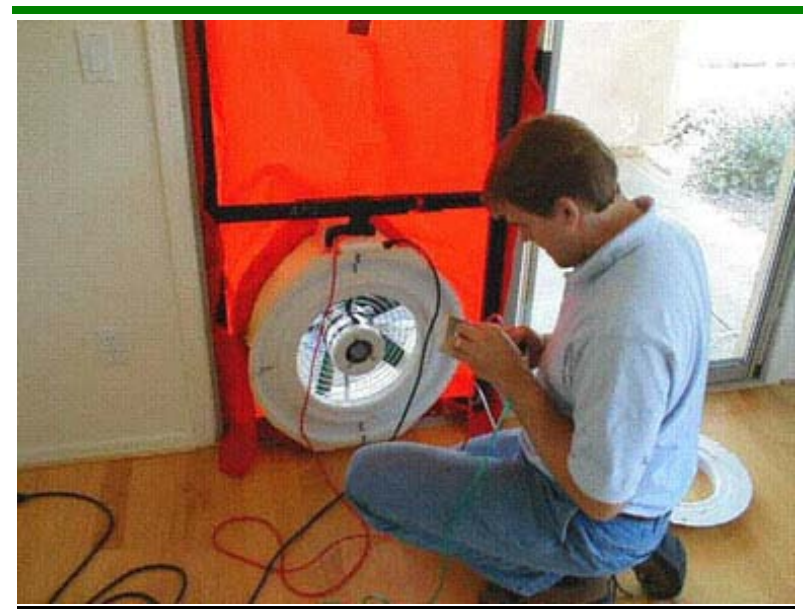

(Arizona Department of Energy, http://www.azenergy.gov/)

Purpose: Tests for air leakage between the interior and exterior of a home, which can lead to significant heating and cooling energy losses and unwanted moisture.

Basic Concept: A powerful calibrated fan in an external doorway sucks air out of the house, which creates a pressure differential that draws external air back in through any cracks in the exterior. Using your hands or a smoke pencil, you can detect where air is coming in to identify leakage spots in need of repair. With gauges, you can also quantify the amount of air leakage as a reference to standards or before/after results of work done on a home.

How It Works: Learning how to use a blower door and all other equipment requires hands-on training. The basic steps include:
- Prepare house according to standard conditions;

- Set up the frame and curtain;

- Install the fan and connect it to a digital manometer (which measures pressures);

- Take a baseline reading with the fan cover on, and

- Take a second reading with the fan cover off to determine the extent of air leakage. When the cover is off, you can use a smoke pencil to locate the source of air leaks.

Standards: RESNET's "Mortgage Industry National Home Energy Rating Systems Standards" require blower door testing in compliance with ASHRAE Standard 119, Section 5.1 , which lays out procedures for measuring air leakage area. The RESNET Standards also specify physical conditions that should be in place prior to testing. The Building Performance Institute's (BPI) Building Analyst Professional Standards require blower door testing under many common scenarios in compliance with ASHRAE Standard 62-89, which provides minimum ventilation requirements. The BPI Standards also require certain physical conditions prior to testing and prohibit depressurization tests when airborne asbestos is a risk.

Cost: A typical system will cost $\$ 2,500-\$ 3,000$, not including cases and major replacement parts. Digital manometers are sold separately for about $\$ 700$ to $\$ 800$.

\section{Duct Blaster}

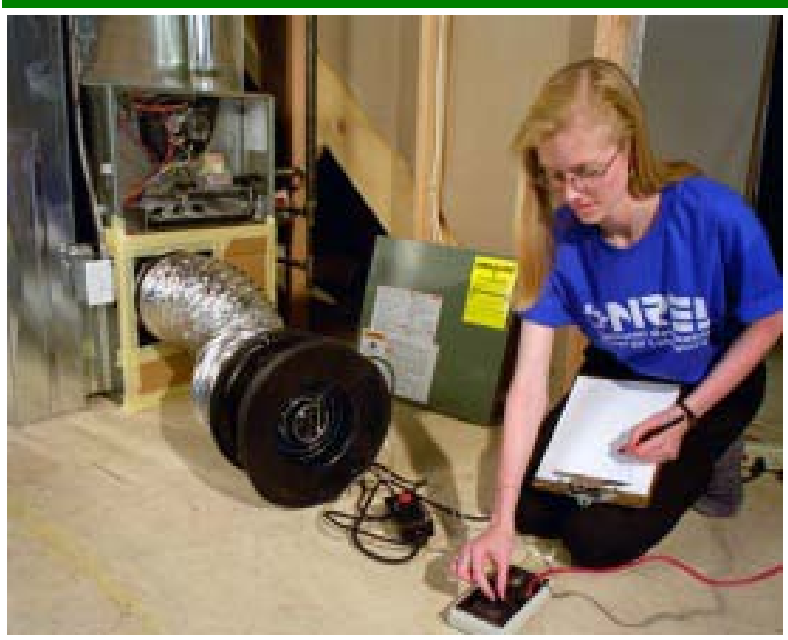

(National Renewable Energy Laboratory/PIX 04913, http://www.nrel.gov/data/pix/Jpegs/04913.jpg) 
Purpose: Tests for air leakage through a home's duct system.

Basic Concept: Whereas a blower door pressurizes an entire home, a duct blaster pressurizes only the home's duct system. Measuring the amount of airflow needed to bring the duct system up to a standard level tells you the amount of air leaking out of the system.

How It Works: Two tests are typically performed with duct blasters: a "total leakage" test that reveals the extent of air leakage throughout the whole system, and an "outdoor test" that measures only leakage to the outside and unconditioned space (which causes energy loss). The basic steps in a total leakage test are as follows:

- The duct blaster is connected to the duct system either at the air handler or a return grille.

- The duct system is isolated from the house by taping off all registers and grilles.

- Indoor and outdoor pressures are equalized by opening an exterior door or window.

- The duct blaster is run, and the airflow required to achieve standard pressurization is measured to determine total leakage.

An "outdoor" test uses the following modified procedure:

- When possible, any unconditioned space in the house is equalized with the outdoors by opening a window or door (if available), and interior access to this space is closed off.

- A blower door and a duct blaster are used simultaneously to equalize the pressure inside the home's conditioned space and inside the duct system.

- The airflow is measured. At this point the only duct system leakage that will occur will be to the outdoors or to unconditioned space because there is no pressure differential within the conditioned space.

Standards: The RESNET Mortgage Industry Standards require that duct leakage testing comply with ASHRAE Standard 152, which establishes procedures for measuring both outside and total duct system leakage. The RESNET Standards include just a few modifications (e.g., supply and return leakage can be measured together). BPI Building Analyst Standards require "before and after" duct leakage testing whenever duct sealing is conducted, but the Standards do not call for specific testing procedures.

Cost: Typically about $\$ 2,000$, plus a digital manometer for about $\$ 700$ to $\$ 800$.
Infrared Camera

Purpose: Tests for missing insulation and air leakage in the building envelope.

Basic Concept: The amount of infrared radiation emitted at different points along the building envelope varies with differences in surface temperature, which can be affected by missing insulation or air leaks. The camera translates the pattern of infrared radiation emitted into a visible image on a screen, using different shades or colors to depict where temperatures diverge.

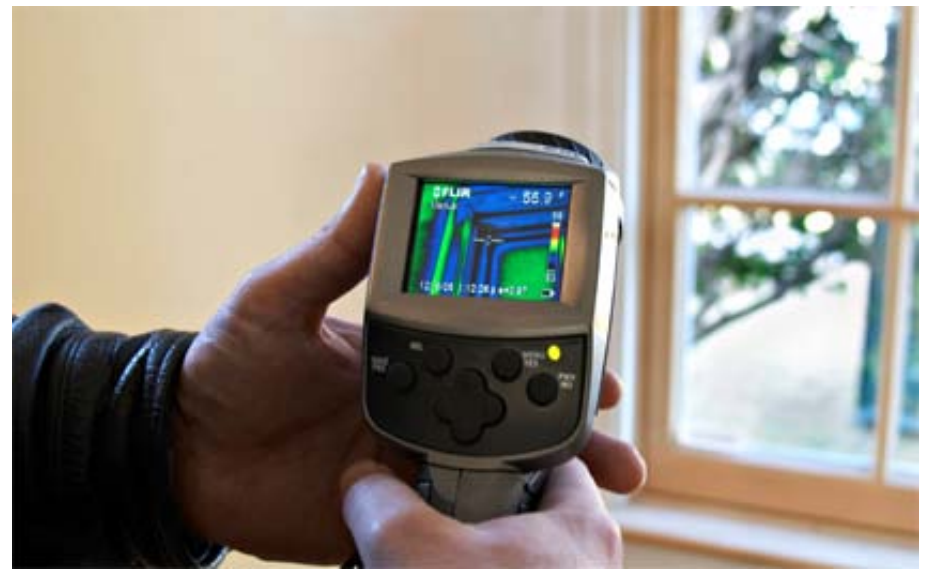

(ENERGY STAR, http://www.energystar.gov/index.cfm?c= behind_the_walls.btw_inspection)

How It Works: Actual operation of an infrared camera is relatively simple, yet interpreting the images displayed can be fairly complex. The most difficult aspects of the process are ensuring that the right physical conditions are in place so that temperature variations will show up properly, as well as being aware of any structural issues that may obscure or impact surface temperature readings.

The temperature difference between the interior and exterior surfaces must be great enough that the variation is apparent in places where there are insulation gaps.

Surface temperature can be affected by all of the following factors:

- Air temperature (both inside and outside)

- Sunlight

- Moisture

- Wind

Keep in mind that temperature changes throughout the day, so it is best to conduct the analysis at a time when the temperature is relatively constant. In addition, the effects of solar gain can affect both exterior and interior surfaces and may take several hours to wear off, so if possible it can help to test late at night or early in the morning (or perhaps on a heavily overcast day). 
Testing for missing insulation can be done by analyzing either the interior or exterior of the home. Temperature variation is sometimes easier to detect indoors, but indoor testing brings its own challenges. Furniture and wall hangings can cause obstructions and should be moved if possible. Other construction elements such as ductwork, radiant heating components in the walls, or internal air cavities may also obscure readings, so knowledge of the building design can be essential. In addition, certain interior spaces may be inaccessible, in which case additional outdoor imaging may be helpful.

When testing for air leakage, it is best to use a blower door to depressurize the home and draw in external air, which will show up as thermal variation if the indoor surface temperature is different. The blower door should be run long enough to change the surface temperature at the point of the leak. Note, however, that running the blower door for too long can eventually minimize the temperature difference of the whole surface with the outdoors. In addition, be aware that if the air passes first through an internal cavity, its temperature may change before it reaches the wall surface, meaning the leak may not show up.

Standards: RESNET recently adopted standards for thermographic testing with an infrared camera to detect both insulation defects and air leakage. The Standards establish a list of required camera specifications, including standards for thermal sensitivity, spectral range, field of view, and image recording. When testing for insulation gaps, the RESNET Standards require an indoor/outdoor temperature differential of $10^{\circ} \mathrm{C}\left(18^{\circ} \mathrm{F}\right)$ for four hours prior to testing, as well as removal of furniture and other obstructions. The temperature differential can be less for air leakage inspections, but blower door usage is required. The RESNET Standards are based on several previously established standards adopted by various organizations in the United States and abroad. BPI has not adopted thermographic inspection standards at this point.

Cost: Prices can vary widely, but cameras that meet the RESNET specifications are available for less than $\$ 10,000$.

\section{Combustion Analyzer}

Purpose: Tests the gas concentrations, temperature, and draft pressure in appliances that burn fuel to determine efficiency, safety, and emissions levels.

Basic Concept: Higher levels of $\mathrm{CO}_{2}$ and lower levels of $\mathrm{CO}$ are safer and indicate efficient fuel combustion with oxygen in the supply air, although too much supply air can cool the combustion system and reduce efficient heat transfer. The analyzer tests for these concentrations along with levels of regulated emissions gases.

Temperature is measured because excess heat leaving through the exhaust flue amounts to inefficient energy loss. Draft is regulated since low draft pressure can cause combustion gas buildup and venting into the home, while high draft pressure can draw too much heat, damaging flames, and explosive gas into the flue.

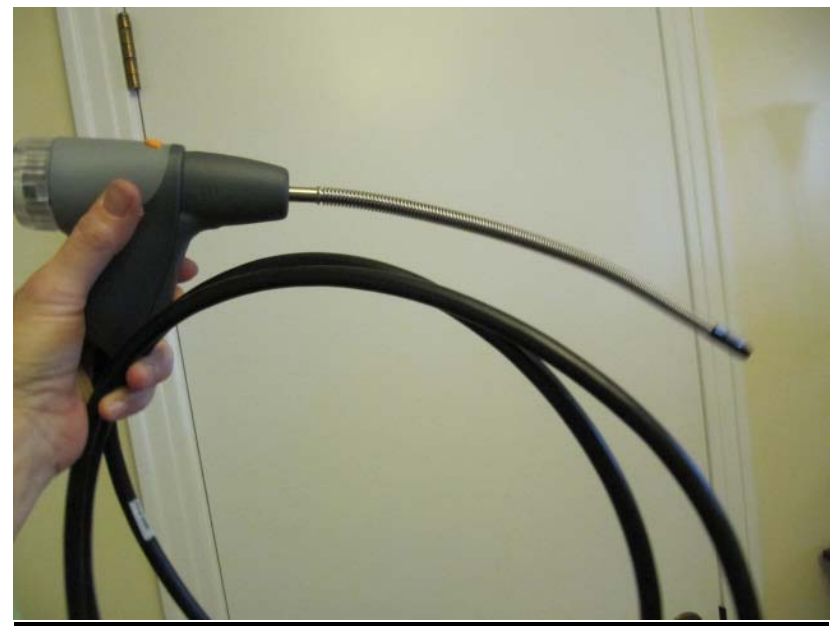

(Lawrence Berkeley National Laboratory, http://homeenergypros.Ibl.gov/ photo/flexible-probe-for-our-testo?context=user)

How It Works: The analyzer determines gas concentrations by measuring electrochemical reactions in a gas sample drawn from the appliance with a probe and pump. Temperature is measured using thermocouples that produce a certain voltage, depending on the temperature difference between two ends of the conductor. Draft pressure is gauged with a manometer or transducer that emits a given signal, depending on the amount of pressure exerted on it.

It is extremely important to receive proper training before conducting any combustion analysis test. The basic procedures that should be covered are as follows:

- Take a gas sample by inserting as close to the base of the flue as possible and below any draft damper, hood, or diverter.

- Measure temperature by placing a thermocouple where the exhaust is hottest, generally at the flue base or just downstream of a heat economizer. Supply air temperature (measured outside the equipment or in the duct system) is subtracted from exhaust temperature to determine the net exhaust temperature.

- Gauge draft pressure with a manometer or transducer, generally at the same point at which the temperature was measured. An additional 
measurement should be taken downstream of any draft damper, hood, or diverter.

The measurements are displayed as readings on a portable electronic screen.

An important aspect of combustion testing is the "worst case" combustion appliance zone (CAZ) test, which gauges potential back-drafting of toxic or explosive gases into the home. Back-drafting is caused by anything that depressurizes the home, such as bathroom fans, kitchen range hoods, clothes dryers, and even unintended duct leaks. A worst-case CAZ test involves taking a pressure reading with all these appliances running (including the air handler) to determine whether the pressure exceeds minimum safety limits. During this test, gas spillage is also detected using a mirror or smoke pencil to determine whether the length of time that gas spills from the appliance after startup is below acceptable limits.

Standards: BPI Standards include very specific requirements regarding combustion analysis testing. The Standards call for $\mathrm{CO}$ measurement, draft measurement, and spillage evaluation, as well as worst-case CAZ testing. Acceptable limits are spelled out for all of these tests, and specific actions are required at certain levels or when the equipment fails to meet specifications. In addition, a clear step-by-step procedure is laid out for combustion safety testing. RESNET Standards on combustion analysis testing were recently adopted and cover gas leakage, worst-case $\mathrm{CAZ}$ depressurization, and $\mathrm{CO}$ testing, although BPI procedures may be substituted by BPIcertified professionals under the RESNET Standards.

Cost: A combustion analyzer can be purchased for $\$ 300$ to $\$ 900$, depending on features.

\section{More Assessment Tools}

To round out your home performance assessment and work scope, some additional equipment may be needed, such as the following:

- A flow hood to gauge the amount of air moving through forced air or ventilation vents, which can create airflow and conditioning problems in particular rooms.

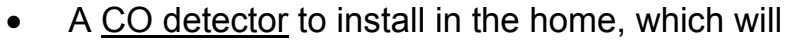
monitor carbon monoxide levels on an ongoing basis.

- A gas sniffer, which checks for natural gas or propane leakage.

- A hygrometer or psychrometer to measure relative humidity, which is key to preventing mold and avoiding moisture damage.
- Appliance meters to determine how much energy is being drawn by major appliances during a sampling period, which can be extrapolated over a year with additional information from the homeowner.

- A digital camera to display what you have found in the attic, crawlspace or other hard-to-reach areas to the homeowner.

\section{Software}

Energy and building software serves as the foundation for the house model, analysis, and recommendations. By inputting your actual measurements and comparing potential efficiency measures, you can recommend a costeffective scope of work to the homeowner.

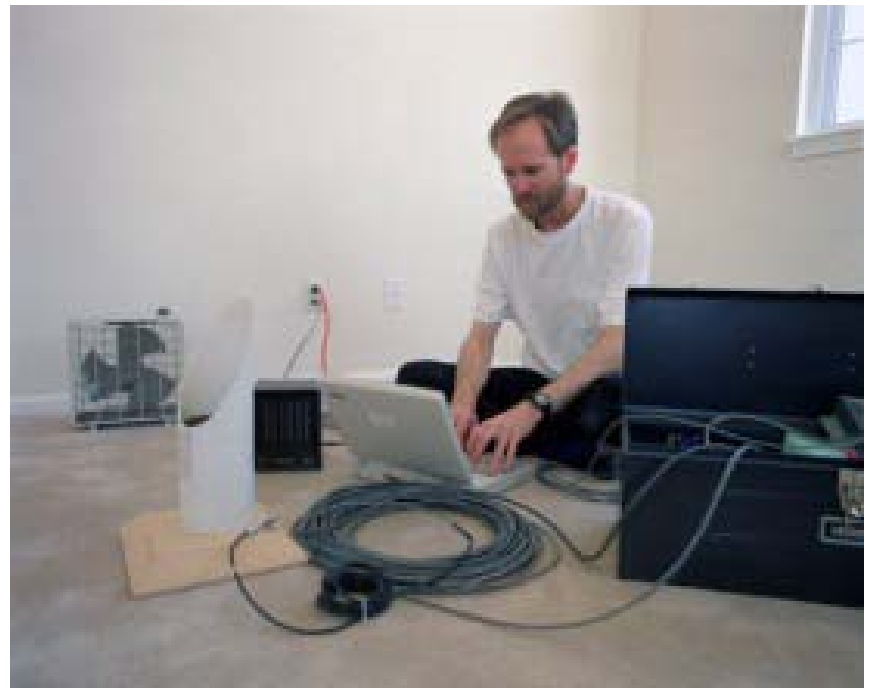

(National Renewable Energy Laboratory/PIX 04866, http://www.nrel.gov/data/pix/Jpegs/04866.jpg)

Strive for the highest level of accuracy of predictions you can afford to ensure credibility. True up with the homeowners' past utility bills. Some new tools achieve impressive levels of accuracy with minimal inputs.

Good software should produce results in a graphic-rich, customer-friendly format. Spend the time necessary to educate the customer on the report so that they understand the scope of work you have proposed. Print out and leave a report behind, if possible.

Software should also allow you to provide a rating or label for the home, both as is and after recommended improvements. This simplified quantification can serve as a powerful means of boiling down lots of detail into a single understandable metric that the homeowner can digest. 
Installation: Cellulose Blower

Purpose: Fills wall cavities, attics, and other spaces with cellulose insulation, increasing the $\mathrm{R}$-value and reducing air leakage.

Basic Concept: Cellulose insulation is made from recycled paper products such as old newspapers and is treated with fire retardant. It is "loose fill" in the sense that it is separated into small chunks by agitators in the cellulose blower drum, which makes it a great option for filling up insulation gaps. Using the right filling technique, it can be densely packed into wall cavities when blown in with a tube, which maximizes the R-value and air sealing effectiveness.

How It Works: Insulation works best when air is not moving through or around it. So it is very important to seal air leaks before installing insulation to ensure the best performance from the insulation.

When installing blown cellulose, particularly in exterior walls, it is essential to be aware of safety concerns such as the location and type of electrical wiring and the potential presence of lead or asbestos. It is also important to understand details of construction such as the integrity of the wall framing, especially when dense packing.

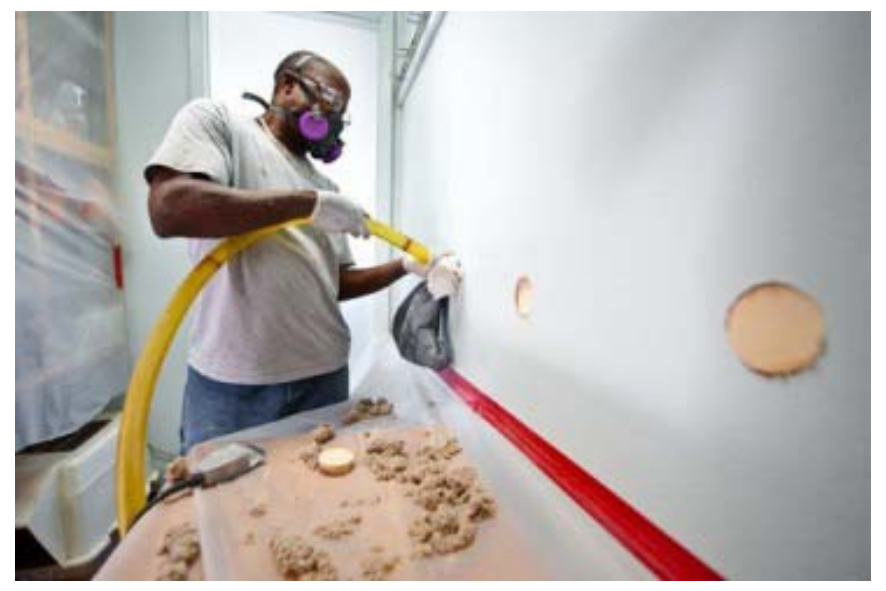

(National Renewable Energy Laboratory/PIX 17964, http://www.nrel.gov/data/pix/Jpegs/17964.jpg)

Be sure to receive proper training before attempting to blow cellulose. The steps that should be covered include the following:

- When insulating existing exterior walls, holes must be drilled in each stud bay (the cavity marked off by the wall studs). If insulating from the outdoors, a portion of the siding or other materials such as shingles must first be removed before the hole can be drilled. Angling the hole can make it easier to slide the tube in a particular direction.
- Cellulose is emptied from its packaging into the blower drum, where it will be broken up by agitators when the blower is turned on. A slide gate controls the rate at which the cellulose is blown in, while another control may regulate the amount of air that flows in simultaneously.

- A tube connected to the blower drum is inserted into the drilled hole. Dense packing can be achieved by sliding the tube first all the way to the top of the cavity and then pulling it back down slightly to leave room for the cellulose to blow in easily. Running only air through the tube first will straighten it out.

- The cellulose blower is switched on manually or with a remote control. To dense pack, the cavity is filled gradually from the top down, removing the tube slowly down toward the drilled hole as it becomes snug along the way.

- The process is completed by clearing the tube and knifing it downward from the hole to the bottom of the cavity, then blowing and gradually removing the tube back up toward the hole.

- The process is repeated for each cavity. When the blowing is complete, the holes are sealed off and any siding or shingles are replaced.

The process for blowing cellulose into the attic is relatively simpler. The key is to measure the floor space so you will know how much insulation is needed and then mark the desired depth on the attic framing. Cellulose bags will typically indicate how many are required to reach a certain R-value. Before blowing any insulation into an attic, first complete all air-sealing work and isolate potential fire hazards, such as recessed lights and bathroom fans. The cellulose can then be blown in directly onto the attic floor, taking care not to cover soffit vents, which can be protected with paneling. Placing a barrier around the attic opening and any other vertical breaches will ensure the cellulose builds up in these areas to the desired depth. After blowing is complete, check with a measuring stick to ensure that depth has been reached. Raking may be required to achieve an even spread.

Standards: BPI's Building Envelope Professional Standard calls for a thorough pre-inspection to identify unsafe or unsound areas for installing insulation, such as areas with knob and tube wiring, recessed light fixtures that have not been rated for direct contact with insulation, areas with moisture problems, and areas that are structurally unsound. At the same time, the Standards actually require installation of dense-packed or foam insulation to block air leakage paths in enclosed cavities, 
with the specification that cellulose must be installed at 3.5 pounds per cubic feet or greater. Refer to this Standard for additional requirements.

RESNET's Contractor Work Scope Reference Standards cite ASTM Standard C1015-06, "Standard Practice for Installation of Cellulosic and Mineral Fiber Loose-Fill Thermal Insulation." This Standard also requires a preinspection to locate and block off certain areas, and it prohibits installation around metal flues, chimneys, or fireplaces. Reviewing the full details of this Standard carefully is strongly recommended.

Cost: Several blowers are available in the range of $\$ 2,500$ to $\$ 5,000$, although more expensive models can be purchased with additional features and higher capacities.

\section{Spray Foam}

Purpose: Spray foam insulation is typically used to insulate walls and prevent air leakage. Dense spray foam known as "closed-cell" insulation also acts as a moisture barrier.

Basic Concept: Two components, a base and a foaming agent, are mixed and sprayed into open wall cavities, or into enclosed spaces using "slow rise" foam. The foam expands when it is sprayed on, filling in any gaps and crevices to create an airtight seal.

How It Works: Two types of sprayed foam are available on the market, known as "open cell" and "closed cell" foam. This refers to the density of the foam: open cell is about 0.5 pounds per cubic foot, while closed-cell is about 2 pounds per cubic foot. Closed-cell foam has a higher Rvalue and may be a better choice if moisture is a concern, although it is typically more expensive.

Depending on which product is used, the closed cell or open cell foaming agent is mixed with the base by a machine that controls mixing rates, pressure, and temperature. Mixing correctly is important because poor mixing has been reported to cause foam to contract over time. This can leave gaps between the foam and the framing that can lead to significant energy loss and can compromise the value of the insulation.

Before applying foam to open cavities such as basement walls with open stud bays, certain areas may need to be protected. These may include any electrical outlets, as well as nearby HVAC equipment, windows or doors, and flooring, as the foam tends to overspread and stick. When filling open cavities, you should wear protective gear such as a polyethylene suit to prevent skin exposure and a respiratory mask with an organic vapor cartridge and particulate filters.

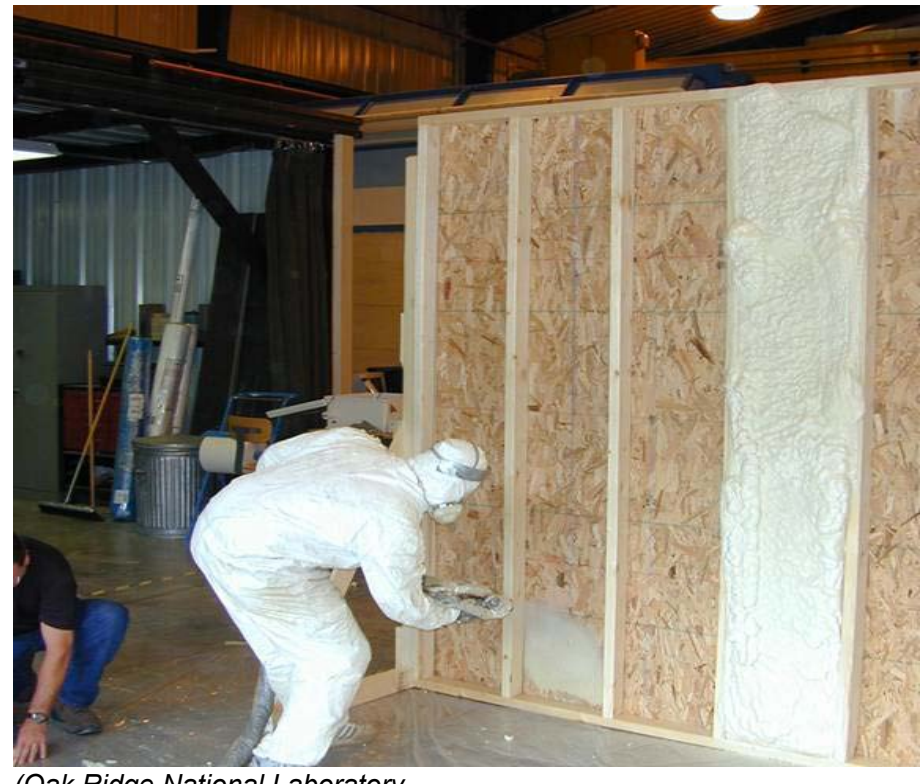

(Oak Ridge National Laboratory,

http://www.ornl.gov/sci/roofs+walls/AWT/HotboxTest/WoodFrame/ SoyBean/index.htm)

When spraying in an open cavity, the foam should be applied evenly between gaps. The foam will expand significantly, so a conservative application should be enough to achieve the desired depth. After the foam has set, a long handsaw blade can be used to shave off the excess foam and make it even with the studs.

With existing walls, installing spray foam requires many of the same considerations as blowing cellulose, including knowing the wall's structural integrity and investigating the location of any electrical outlets, wiring, or obstructions in the wall. To install the foam, holes should be drilled in the wall cavities and a tube inserted into the hole. Multiple tubes may be required for the job because the foam may "cure" (expand) inside the tubing. Drilling multiple holes and filling from the bottom up can help to ensure an airtight seal with no gaps. Once the holes are drilled, foam should be sprayed slowly into the enclosed cavity and given a few minutes to expand. If excess foam emerges from the holes, it can be shaved and sanded down prior to patching the holes.

Standards: Many of the same requirements that apply to cellulose blowing under the BPI Envelope Professional Standard are also applicable to spray foam insulation. In addition, the Standard requires that spray foam be installed at or above the manufacturer's recommended density to limit airflow. The RESNET Standards do not specifically cite any reference standards for installing spray foam. ASTM Standards dictate certain manufacturer specifications, as do certain national and international code organizations. 
Cost: Prices are highly variable, as a full professional system may require multiple components that are often sold separately.

\section{Resources}

DOE Building Energy Software Tools Directory. http://apps1.eere.energy.gov/buildings/tools directory/subj ects sub.cfm

Home Energy Magazine: "So You Want to Be a Home Performance Contractor" (4/16/2006).

http://www.homeenergy.org/show/article/page/7/id/199

RESNET Mortgage Industry National Standards. http://www.resnet.us/standards/RESNET Mortgage Indus try National HERS Standards.pdf

RESNET Guidelines for Thermographic Inspections of Buildings.

http://www.resnet.us/standards/RESNET IR interim guid elines.pdf

Building Performance Institute Standards.

http://www.bpi.org/standards.aspx

ASTM Standards.

http://www.astm.org/Standard/index.shtml

"Air Seal and Insulate with ENERGY STAR."

(http://www.energystar.gov/index.cfm?c=home sealing.hm improvement sealing)

"Independent Testing and Inspection to Ensure Quality Construction," ENERGY STAR.

http://www.energystar.gov/index.cfm?c=behind the walls. btw inspection

"Flexible Probe for Our Test 327 Combustion Analyzer." http://homeenergypros.lbl.gov/photo/flexible-probe-for-ourtesto?context=user

"Advanced Wall Systems: Hotbox Test R-value Database." http://www.ornl.gov/sci/roofs+walls/AWT/HotboxTest/Wood Frame/SoyBean/index.htm 
\begin{tabular}{l|l} 
u.s. department of & $\begin{array}{l}\text { Energy Efficiency \& } \\
\text { Renewable Energy }\end{array}$
\end{tabular}

\section{Appendix D - Customer Education}




\section{HVAC Transition Guide for Building Performance Contractors}

\section{Operational Area: Marketing and Customer Contact}

\section{Work Activity: Customer Education}

\section{Issue}

Customer education is essential to generating business as a home performance contractor. Crafting a clear and concise message about the benefits of your services and spreading that message effectively will greatly increase your potential customer base.

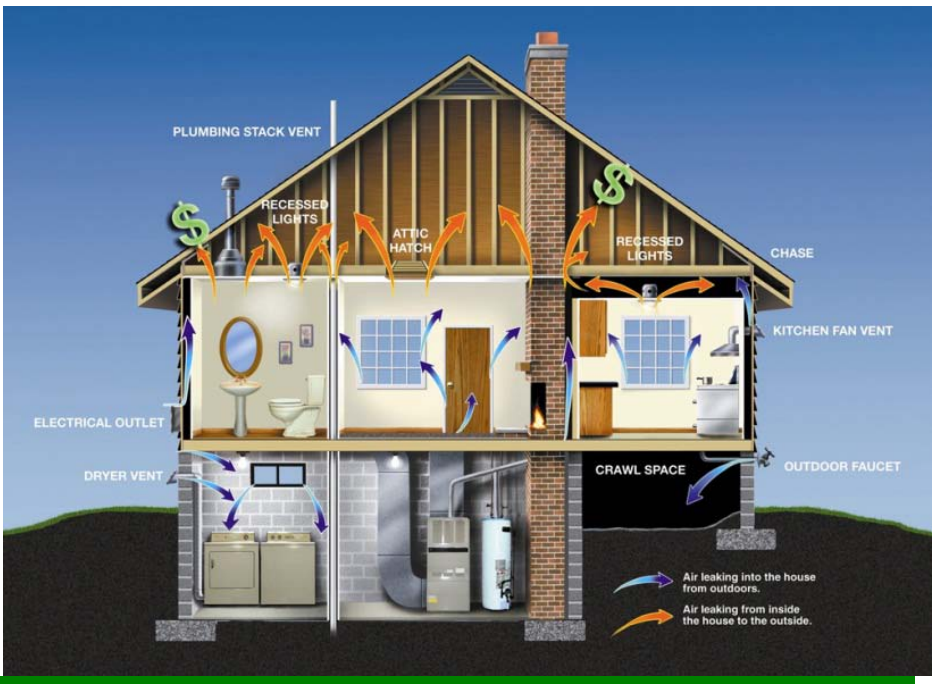

(ENERGY STAR,

http://www.energystar.gov/index.cfm?c=behind_the_walls.btw_airsealing)

\section{Best Practices}

There are many ways to educate customers about the benefits of your services. The key is to frame the message in a way that grabs customers' attention and then to follow through with additional information on your comprehensive offerings. Consider the following "best practices" when devising your own customer education strategy:

- Describe yourself as a problem solver. The best way to get in the door is to offer a solution to a problem that many potential customers are having, such as comfort issues, mold and mildew, or high energy bills. Once you've been invited inside, you can explain more about the value of a whole-house assessment.

- When using media to attract customers, remember that the most important thing is to "break through the clutter" of too much advertising. Short, attentiongrabbing headlines can be at least as important as the rest of the message. You should also display in ads and marketing materials any certifications you have acquired to enhance your professional credibility.

- Sometimes the best way to deliver your message is through others. Referrals are especially powerful because customers learn about your business from someone they already trust. A good way to get referred is to go out of your way to satisfy your existing customers. Word of mouth can spread quickly in a neighborhood when one customer is happy.

- You can generate additional referrals by making presentations. Start by presenting to relevant groups such as home inspectors and real estate agents, who may be asked by their own customers for contractor recommendations. Business groups and professional associations may also be interested in learning more about the services you provide.

- Presenting to licensed or certified housing professionals who require regular continuing education unit (CEU) credits can be an effective means of spreading the word about you and your business. Real estate agents, architects, appraisers, and others are always looking for new CEU topics and presenters. With a little investment in development of a curriculum and completion of an application, many state licensing boards and associations will welcome a new home performance class.

- Home shows tend to draw a lot of homeowners looking for new ideas, products, and services. Purchase a booth in a prominent location, display your diagnostic tools, including your blower door and infrared camera, as conversation props, and talk to a lot of consumers. Spread the word on your business and its benefits.

- Don't overlook existing customers. Now that you offer a wider range of services, customers who already know you may be open to learning more about the benefits of your expanded business because they will already have a general understanding of what you do. Plus, customer retention typically costs far less than new customer generation. 
Tools

A number of different tools can be used to educate customers (or those who might provide referrals) about the benefits of your business, including the following:

- Direct Mail: Make sure to craft a well-thought-out list of customers to target. Current customers and potential customers in a similar demographic or neighborhood are a good place to start.

- $\quad$ Internet: Building a website is no longer enough. Work with your web designer to make sure it's built so that it will show up in search results. Consider creating Facebook and Twitter accounts and perhaps a weekly blog with home performance tips.

- Email: Use your website to collect "email sign-ups." Then send out newsletters or helpful tips that potential customers will actually want to read.

- Traditional media continues to be an effective way of reaching customers, including the following:

- Print media, such as

- Newspapers

- Magazines

- Trade publications

- Radio/Television: Consider both advertising and being interviewed on talk shows (or even creating a talk show of your own related to home performance)

- Handouts and Specialty Items: Items that provide your web address and contact information can be sent or handed to potential and existing customers, encouraging them to learn more about the solutions you offer when a relevant problem arises. The best items are those that are likely to stick around and be seen often or when it matters most (e.g., refrigerator magnets, thermostat stickers, and valve tags).

- $\quad$ Customer testimonials are an effective way of backing up your own statements about your business. Video testimonials on your website can be especially powerful.

\section{Article Snippets}

Home Energy Magazine:

Marketing Essentials

Five leaders in the home performance field-Greg Thomas, Dick Kornbluth, Larry Taylor, Darin Hughes, and Carl Seville-reveal how they get their phones to ring.
- "If you offer multiple services, market all of those services in the same venues that you have always marketed your individual services, but tweak your message. For example, if you have traditionally advertised in a local newspaper's special fall home issue, then continue to do so; just make sure to mention specific benefits that you can provide that homeowners need. If there are specific climaterelated problems in your area, such as ice dams in the winter or cooling comfort problems in the summer, market your ability to provide solutions to those problems."

- "Work hard to generate referrals by taking good care of your customers. You can also get leverage in educating customers through delivering presentations. We once did a presentation that only one person attended. But he was a home inspector. And we got a steady stream of referrals from that inspector for years after." 4/16/2006 http://www.homeenergy.org/show/article/nav/salesandmarketing/pag e/2/id/200

\section{A Whirlwind Startup}

Mike Woodson and Jerold Sit, principals of Practically Free Energy:

- "They bought some advertising airtime on a local radio station and talked their way into a monthly show with one of the station's regular hosts.... 'It can be like giving a haircut over the phone,' says Woodson. When discussing building science principles on air, Woodson sticks to easy-to-understand metaphors. 'We don't talk about CFM and convection,' he says. 'We talk about filling up a house with basketballs. We talk about heat transfer in terms of hair dryers and frying pans."' 3/3/2006 http://www.homeenergy.org/show/article/nav//id/148

\section{Contractor Excellence:}

\section{Solid Marketing Strategies for a Struggling Economy}

"You need to educate [customers] as you go or they won't understand the justification. It's like when you see a chugging, un-tuned old car rumble past and think, 'Wow, for the gas they're wasting every few miles, they could have their car tuned up.' In other words, the service would pay them a profit, but they've not been taught. That's your job." ("Solid Marketing Strategies for a Struggling

Economy") 7/1/2008

http://www.contractorexcellence.com/967 
Home Energy Magazine Archive:

http://www.homeenergy.org/

ACCA Contractor Excellence Marketing Column:

http://www.contractorexcellence.com/author/ahudson

Air Sealing and Insulation That Works, ENERGY STAR,

http://www.energystar.gov/index.cfm?c=behind the walls.

btw airsealing 


\section{Appendix E - Community Engagement}




\section{HVAC Transition Guide for Building Performance Contractors}

\section{Operational Area: Marketing and Customer Contact}

\section{Work Activity: Community Engagement}

\section{Issue}

Engaging the community in efforts to save energy carries not only public awareness of this important issue but can also increase demand for your home performance business. As a contractor, you can reach out to community members on your own or by partnering with public agencies and community groups. Either way, a good outreach strategy will put you at the top of customers' minds when they decide to make efficiency improvements to their living environment.

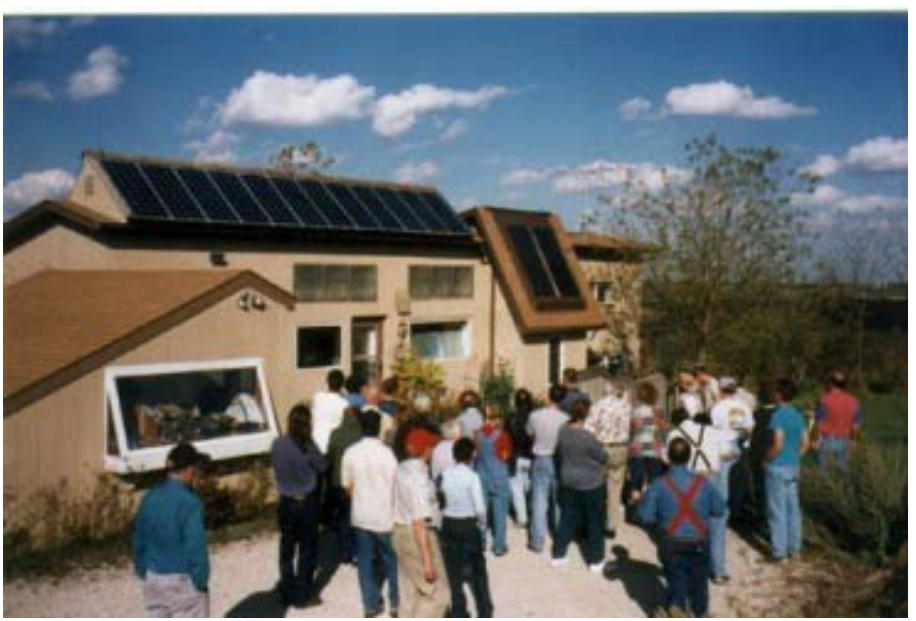

(Photo courtesy of National Renewable Energy Laboratory/PIX 10520, http://www.nrel.gov/data/pix/Jpegs/10520.jpg).

\section{Best Practices}

Effective community engagement strategies can vary widely. The focus of any strategy, however, should first be on getting people's attention, then delivering your message through effective channels, and finally following through to turn heightened awareness into action. The following tips will h`elp you implement your strategy:

- $\quad$ Focus on what really motivates people. Reducing greenhouse gas emissions is good, but often individuals have other motivations such as saving money or fixing home performance problems. Lead with whatever message causes people to pay attention.

- Advertise your services at community events, and arrange speaking engagements in front of groups of people. Events such as home shows, community fairs, and home-improvement workshops are useful for reaching people who may already want to do something in their home. Speaking at neighborhood venues will give people a chance to discuss the information you present with people they trust.

- Establish partnerships with public programs and community organizations that are running energy efficiency campaigns. Often these programs will have lists of qualified home performance contractors who are able to carry out energy efficiency work.

Sometimes they will also provide subsidies for home performance assessments and contracting work, lowering the cost of your services to community members.

- $\quad$ Reach out to neighbors in the areas where you are working. Even before you contact them, they may have seen the work going on and may be curious to learn more. You can provide information using flyers and mailings or go door-to-door in the project area, but be aware that some neighbors may view an unsolicited knock as an intrusion.

- $\quad$ Rate and label the homes you upgrade with before and after scores so that the homeowner is left with a simple quantification of the energy improvements made to the home. This will help demonstrate the impact of your work and will allow the homeowner to boast to their neighbors.

- Be sure to follow up with additional communication after conducting initial outreach. Collect contact information from all your community interactions, and reach out by email, telephone, or regular mail to provide additional information. Getting community members to commit to something as complex and capital intensive as home performance improvement requires continued engagement over a period of time.

\section{Tools}

Some of the best tools for community engagement can be the simplest, such as the following:

- Before you start working on a customer's house, place door hangers on the doors of your customer's neighbors. The hangers can provide information about all the benefits your customer is receiving from your services, which may encourage the neighbors to make similar improvements to their own homes. 
- After you have completed a job, give your customer several "Pass It On" cards to share with neighbors and friends. That way, when your satisfied customers are asked about their experience, they can do your advertising for you.

- Direct mail can also be a very effective tool, but make sure to create a targeted list. Direct mailing a whole community can be costly and tends to yield low response rates.

While these methods can be very effective, higher tech community engagement tools can sometimes be more versatile. For example:

- Through your website, you can provide basic information about your services and can engage people actively with frequent updates. Consider posting home performance tips and creating a forum so users can share their own tips and experience.

- Blogging and social networking are great ways to get community members involved as well. You can ask customers to describe their experience on your blog, and you and your customers can share performance tips through your Facebook or other social networking site.

\section{Article Snippets}

\section{Action Research:}

- "Our review of the most successful projects indicated that word-of-mouth (friends, neighbors, and community leaders) serves an important means for learning about and encouraging program participation.... Importantly, industry leader interviews offered an important caveat pertaining to word-of-mouth approaches. Specifically, they warned that word-of-mouth does not begin until the first contractor is paid and the homeowner is happy with the work."

- "[Focus group] participants were positive about the potential community-level benefits of utilizing a whole house approach and were less motivated by individual "green" labels or ratings.... Participants described the normative effects of such improvements, stating the potential "domino effect." They commented that such changes would ultimately improve their whole neighborhood."

- "Participants consistently mentioned wanting to talk to real homeowners who had been through a home performance experience, as well as being able to see the results. Neighbors were considered to be a highly reliable source of information not only because they were trustworthy and not profiting, but because presumably their home is similar in size." http://www.builditgreen.org/ files/DevCom/Greenpost/CBSM Report .pdf

Lawrence Berkeley National Laboratory:

Driving Demand for Home Energy Improvements

- Houston's Residential Energy Efficiency Program (REEP) weatherization program has found that using contractors to market the program while they are working in a neighborhood is a very effective outreach technique. When the contractors weatherize a home, they visit neighboring homes and talk to residents or leave door hangers. These residents can then reach out to talk to their neighbor who just got her home weatherized to confirm that they had a positive experience. http://drivingdemand.Ibl.gov/

\section{Clean Energy Solutions:}

- Make use of traditional community organizing tools... and social marketing through neighborhood networks. In the residential and small commercial sector, doorto-door canvassing is an incredibly effective recruitment and educational tool. http://cleanenergysol.com/news/BPs\%20Marketing\%20Strategies\% 209-3-10.pdf

\section{Resources}

Action Research, "Community Based Social Marketing to Inform Homeowner Participation in California EnergyEfficiency Home Improvement Programs," prepared for Build It Green, July 2010.

http://www.builditgreen.org/ files/DevCom/Greenpost/CBS M Report.pdf

Lawrence Berkeley National Laboratory, "Driving Demand for Home Energy Improvements," September 2010. http://drivingdemand.Ibl.gov/

Clean Energy Solutions, "Best Practices in Community Energy Efficiency Programs," September 2010. http://cleanenergysol.com/news/news EAFrame.html

Department of Energy's "Home Energy Score" rating. http://www1.eere.energy.gov/buildings/homeenergyscore/ methodology.html

EnergySavvy is a simple, free, online home rating tool. http://www.energysavvy.com/estimate/ 
\begin{tabular}{l|l} 
u.s. department of & $\begin{array}{l}\text { Energy Efficiency \& } \\
\text { Renewable Energy }\end{array}$
\end{tabular}

\section{Appendix F - On-Site Customer Interview}




\section{HVAC Transition Guide for Building Performance Contractors}

\section{Operational Area: Assessment}

\section{Work Activity: Onsite Customer Interview}

\section{Issue}

Conducting an onsite customer interview is the first step in diagnosing a home's performance issues. Even without technical expertise, customers often know when something is wrong in their home simply by virtue of spending so much time in it. Listening to the customer attentively can provide invaluable clues that point toward particular problems, which in turn will shorten the time it takes to find solutions.

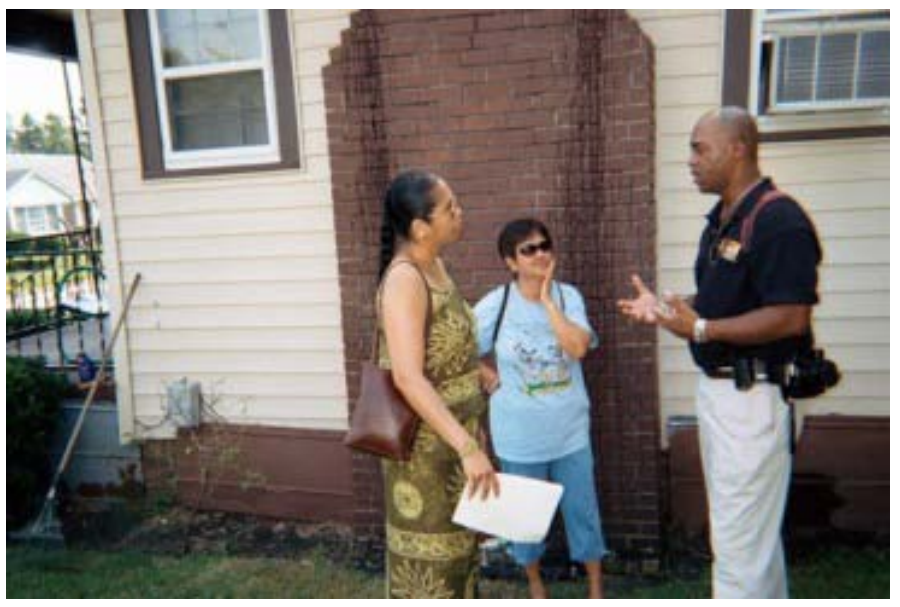

(National Renewable Energy Laboratory,

http://www.nrel.gov/data/pix/Jpegs/09664.jpg)

\section{Best Practices}

The onsite interview is the customer's first real chance to get to know you, so it is important to maintain a professional demeanor and to focus on establishing the customer's trust. In addition, you should listen to the customer with open ears so that you will not miss anything later during the whole house assessment that the customer might point out as an issue. Some tips to keep in mind when conducting an onsite interview include the following:

- Make sure the homeowner is aware of your certifications (BPI, NATE, RESNET, etc.) and understands enough about what they mean to feel comfortable with your level of competency.

- Come prepared. Show up with handouts, fact sheets, and other educational materials relevant to the customer's house. If you have received their utility data already, pre-load it into your energy analysis software to enable you to show them results, costs, and savings before you leave.

- Present yourself as a professional. Show up on time, communicate regularly, manage expectations, take off your shoes, clean up afterward, fulfill your promises, and, generally, leave a positive impression that your work will follow suit.

- Make eye contact, and pay attention when the customer is talking. Avoid jumping in to disagree or debate with the customer, even if you can think of an alternate explanation for a problem. Give the customer a chance to explain any issues fully from their perspective.

- Gather as much information as possible in the interview, and listen for clues that might reveal potential problems.

- Ask follow-up questions to make sure you understand what the customer has told you, and delve more deeply into any potential issues that you flag.

- Refrain from forming conclusions too early about the customer's home performance issues, and avoid guesswork. Keep an open mind until you have finished the interview and have had a chance to conduct a full assessment.

\section{Key Questions}

Asking the customer some basic questions will provide a starting point for a whole house assessment. Identifying problem areas, usage patterns, and the customer's own objectives will streamline the assessment process before it begins and will give you a sense of what to focus on. The following set of essential questions should be asked of most homeowners during onsite interviews:

- Problem Areas:

- Do any rooms in the house feel too hot or too cold?

- Are any rooms or areas of the house especially drafty?

- Are there any signs of excess moisture, mold, or mildew inside the house?

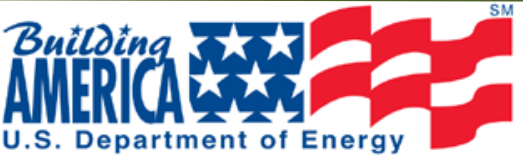


- Are any mechanical systems (HVAC, water heating, etc.) or appliances not working properly? If so, what seems to be the problem, and how long has it gone on?

- Does anything else regarding the house's performance look or feel "wrong"?

- Usage Patterns

- How many people live in the house?

- How much time do people spend in the home?

- How many showers are taken daily and for how long?

- Which rooms in the home are used frequently/infrequently?

- What are the typical thermostat settings?

- When were the mechanical systems and major appliances in the home installed or replaced? How frequently are they maintained, and when did they last receive maintenance? Are there any maintenance records?

- Are there any unusual energy-using products in the house (waterbed, aquarium, kiln, manufacturing facilities, welding equipment, engine block heaters, etc.)?

- Are there any future plans for the home (additions, renovation/remodeling, more/less time spent in the home, planning to sell, etc.)?

- Client's Objectives

$\begin{array}{ll}\circ & \text { Increased comfort? } \\ \circ & \text { Lower utility bills? } \\ \circ & \text { Increased safety? } \\ \circ & \text { Improved environmental footprint? } \\ \circ & \text { Other? }\end{array}$

In addition, if you have not yet received them, you should ask the customer for at least a year's worth of previous utility bills in order to establish how much fuel of various types the customer is using and at what cost.

\section{Article Snippets}

\section{Contracting Business:}

Be a Detective, and 'Investigate' During Service Calls Tina Coop, service and installation manager, CroppMetcalfe, Rockville, Maryland:

- "A good investigation starts with listening and asking questions. Unfortunately, this is an area in which I believe many technicians are lacking. You must arrive at each home and view it as a blank slate. Listen to the homeowner. Gather every piece of information that you can. Don't form opinions or decide on a course of action until you have all the facts."

- $\quad$ "The trouble is, many technicians aren't paying attention when customers are talking. They think they are the experts and are afraid that asking questions may make them look unknowledgeable in their customers' eyes. Nothing could be further from the truth. Homeowners know their homes. They live there every day, and they know when something's wrong or different. We're only there for a few hours."

- "Another technician and I went to a customer's home, and as we talked about the comfort problems she was experiencing, she mentioned that she never needed to change her air filter: it never got dirty. If we hadn't talked with her, we might have seen a clean filter and assumed she had just changed it. But the filter that never got dirty provided the clue we needed to diagnose a return air duct problem. We fixed the line, cleaned out all the dirt in it that never made it to that nice clean filter, and created a happy customer." $3 / 1 / 2011$

http://contractingbusiness.com/service/be-detective-investigate-0311/

\section{Running Service Step 2: Your First 60 Seconds}

Charlie Greer, creator of Tec Daddy's Service Technician Survival School on DVD:

"While customers are explaining their problems, try not to be walking or doing anything. It's best if you're facing each other, giving the customer your undivided attention, and making eye contact. After you've listened to their response, repeat back to them what they just said. This is called being an 'active listener.' "11/1/2009

http://contractingbusiness.com/columnists/greer/running-service-step-2$\underline{1109 /}$

Two Ears, One Mouth: Listen Your Way to Increased Sales

Charlie Greer, creator of Tec Daddy's Service Technician Survival School on DVD:

- "You can't think and listen at the same time, so thinking about your opinions on what's being said instead of concentrating on what is actually being said will interfere with listening. You can't speak and listen at the same time, so interrupting and talking over others is a problem. Deciding what people are going to say and starting to compose your response before they finish speaking is undoubtedly what's happening when people 
complain that we didn't exactly answer the question they asked."

- You were given two ears and one mouth. Use them in that proportion. 4/1/2011

http://contractingbusiness.com/columnists/greer/two-ears-onemouth-0411/

\section{Resources}

Contracting Business:

http://contractingbusiness.com

Home Energy Magazine:

http://www.homeenergy.org/list/archive

ACCA Contractor Excellence:

http://www.contractorexcellence.com/ 


\section{Appendix G - Whole House Performance Assessment}




\section{HVAC Transition Guide for Building Performance Contractors}

\section{Operational Area: Assessment}

\section{Work Activity: Whole House Performance Assessment}

\section{Issue}

The purpose of a whole house performance assessment is to identify for the homeowner the amount of energy currently being consumed, pinpoint the largest sources of energy use and unnecessary energy loss, and identify a prioritized list of potential energy saving measures, while also considering health and safety. Once identified, these measures should be presented to the homeowner as part of a report that analyzes their cost effectiveness. In addition, the report should focus on the recommended measures' impact in terms of health, safety and comfort, as well as their potential to raise the value of the home.

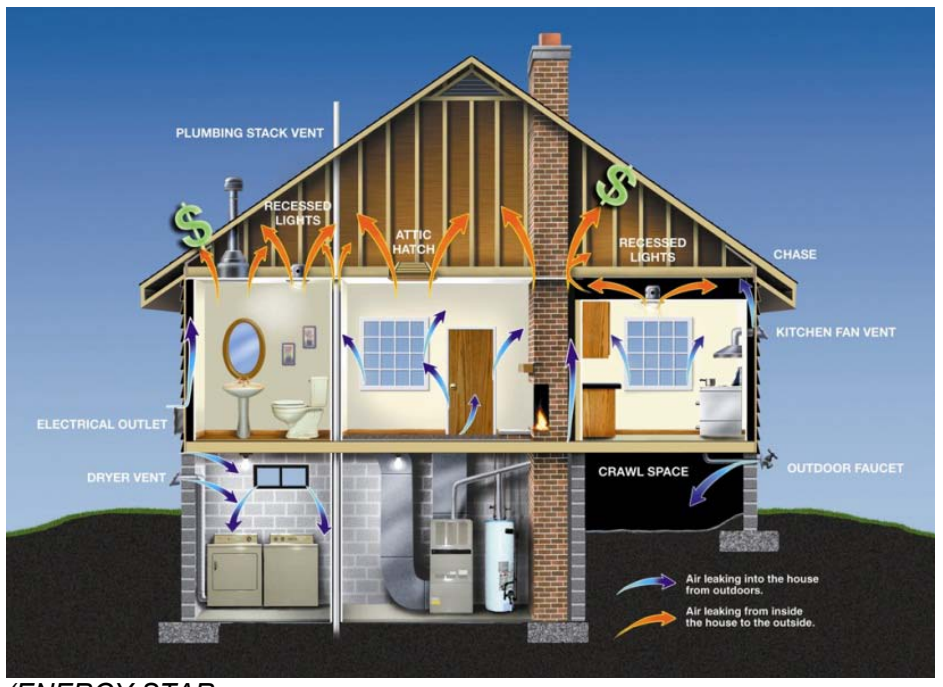

(ENERGY STAR,

http://www.energystar.gov/index.cfm?c=behind_the_walls.btw_airsealing)

\section{Air Leakage}

Air leakage can account for up to 40 percent of the cost of conditioning a home. Finding and sealing air leaks is a top priority in any whole house performance assessment. The most common techniques used for identifying air leaks are as follows:

- Blower door testing. Using a fan and a frame installed in an exterior doorway, the blower door creates a pressure differential with the outdoors so that air leaks can be identified. Feeling with your hands or using a smoke pencil during a blower door test will help you locate the source of any air leaks. A digital manometer hooked up to the blower door can also give you a pressure reading to help you quantify the extent of the home's leakiness.

- Duct leakage testing. Similar in concept to a blower door, a "duct blaster" pressurizes just the duct system of a home to make it easier to identify conditioned air leaks. A "total leakage test" will tell you how much air is leaking out of the system overall. Since only the air leaked into unconditioned space or outdoors causes energy loss, a second "outside test" is run with the duct and conditioned space equally pressurized using a blower door and duct blaster simultaneously. The resulting leakage indicates how much conditioned air is leaving the conditioned space.

- Infrared camera analysis. Often used in conjunction with a blower door, an infrared camera can make it easier to spot the sources of air leaks. Particularly on cold days, outside air that is drawn in will cool surfaces near the leaks, changing the amount of radiation emitted in that spot and showing up on the camera.

In addition to running these tests, it is important to understand how the structural design of the home may increase the chances of air leakage. Ceiling construction, wall and floor cavities and chase-ways, and other structural elements can create built-in air leaks that need to be sealed. Be prepared to recommend sealing strategies such as installing rigid air barriers, dense-pack insulation, foam sealants and weather stripping to prevent further leaks.

\section{Insulation}

Insulation protects the thermal boundary of the home by slowing the rate of heat transfer between the conditioned space and the exterior. While many types of insulation exist, generally they all work by reducing air convection within the insulated space and slowing heat conduction across the boundary. Gaps in the insulation, however, can lead to "thermal bridging," or rapid heat transfer across uninsulated building materials. Finding and filling those gaps is thus an important part of the home performance assessment process.

One of the best ways to find insulation gaps is with the use of an infrared camera, which can detect varying amounts 
of radiation at the surface of the building envelope.

Radiation levels tend to vary with temperature, so

anomalies that show up as different colors or shades may

indicate insulation gaps. Many factors can affect infrared

readings, however, so it is important to learn to conduct

these tests and interpret the results properly.

\section{HVAC}

HVAC contractors are typically well aware of the procedures necessary for checking and tuning up heating and cooling. These checks are an important part of a whole house performance assessment because so much of a home's efficiency level depends on the performance of these systems.

Homes with combustion heating systems should undergo a thorough combustion analysis, not only to assess whether the system presents any safety concerns, but also to determine the level of combustion efficiency. Often heat is lost in the combustion process as a result of excess combustion air, which can be assessed and adjusted through the use of a combustion analyzer. Testing for spillage and backdrafting should also be done simultaneously.

While the steady-state efficiency of the heating system can be noted, the more important measure of the heating system performance is the overall seasonal efficiency, or the Annual Fuel Utilization Efficiency (AFUE). Additionally, the distribution system (typically ductwork or hydronic piping) plays an important role in overall system efficiency. Total system efficiency rating information is included as part of the Residential Energy Service Network (RESNET) "Mortgage Industry National Home Energy Rating Standards".

The efficiency of other heating types should be assessed and reported, as well. For example, in homes with air source heat pumps, the Heating Seasonal Performance Factor (HSPF) should be noted.

Cooling assessments often involve more than simply checking the existing air conditioning system. Many options may exist to reduce the need for air conditioning usage, such as increasing shading and ventilation and applying a reflective roof coating. In terms of the system itself, the Seasonal Energy Efficiency Ratio (SEER) should be noted for central air conditioners and the Energy Efficiency Ratio (EER) for room air conditioners, and these should be compared with the efficiency ratings of newer systems currently on the market.

Installing programmable thermostats can also reduce wasted energy quickly.

\section{Water Heating}

Water heating uses as much as 15 percent of all electricity and 25 percent of all natural gas in the residential sector. The efficiency of water heaters varies significantly by design and capacity. Water heaters are rated for efficiency with an "energy factor", (EF) which is a ratio between zero and one describing the amount of heat input that remains in the water when it leaves the storage tank.

Several efficiency measures can help to improve the efficiency of an existing water heater. These measures should be checked and may be recommended if they have not already been installed. They can include items such as low-flow shower heads, tank and pipe insulation, and automatic temperature controls that adjust to typical demand schedules. Since most water heaters rarely last more than 15 years, replacing an older model with a new more efficient one can both head off a potential future problem when it dies and save energy in the mean time.

\section{Lighting and Appliances}

Lighting and appliances make up a significant portion of a home's total energy consumption, particularly in homes located in milder climates. Lighting itself typically accounts for between five and ten percent of a home's energy costs. A home performance assessment should take note of current lighting usage, including the number of lights in a home, the wattage, and the length of time they are typically left on. Recommendations to improve efficiency may include replacing existing bulbs with more efficiency ones such as compact fluorescents lights (CFLs), as well as installing lighting controls like timers, photo sensors, and occupancy sensors that turn lights off when they are not needed.

Appliances may also require considerable energy consumption, depending on their age, size, type and design. Refrigerators and freezers tend to draw the most energy, followed by clothes dryers, clothes washers, and dishwashers. A whole-house performance assessment should note whether the home's appliances have energy efficiency labels such as ENERGY STAR® and should compare current consumption to theoretical consumption with efficient replacements. The report to the homeowner should also discuss behavioral factors that can impact energy consumption.

\section{Health, Safety and Comfort}

In addition to the systems discussed above, several additional elements of the home can affect the health, safety and comfort of its occupants. These items should 
all be incorporated into a whole-house performance assessment. They may include:

- Indoor air quality: checking for air pollutants, carbon monoxide and radon levels, and biological organisms that can create health problems such as asthma.

- Moisture management: looking for air leaks and condensation and measuring relative humidity with a hygrometer or psychrometer. Moisture problems and excess humidity can damage systems and materials in the home, cause mold, and create occupant discomfort.

- Ventilation: determining whether the home is tight enough to warrant installing a mechanical ventilation system. This can be a simple as recommending the installation of an efficient bathroom fan on a 24-hour timer.

A fully comprehensive home-performance assessment will also assess the occupants' thermal comfort, noise levels, and other related issues that can potentially be corrected to enhance the living experience in the home.

\section{Home Performance Modeling}

Once you have completed your walk-through assessment, you can input the information you have gathered into an energy modeling software program. These programs will allow you to compare the home's current energy consumption and costs with the energy and cost required to operate a theoretical home with your recommended measures installed. You will also be able to rank the measures by cost effectiveness.

A good practice is to bring a laptop with you so that you can input the information onsite and give the homeowner a chance to interactively engage with the software. This will give them a better sense of the value of your recommendations. The best software programs are easily interpreted and present results in a customer-friendly format. You may even want to bring a printer with you so you can leave a copy of the report behind when you depart.

\section{Professional Standards}

Conducting your home performance assessment according to a set of professional standards will ensure that you engage in your work thoroughly and comprehensively, and it will give the homeowner confidence that they are receiving quality service. You should receive training and certification in whatever set of standards you choose prior to advertising your ability to conduct an assessment according to these guidelines.
The two building performance certifications most recognized by state and federal programs today are those issued by the Building Performance Institute (BPI) and RESNET. Historically, BPI focused primarily on existing buildings, while RESNET's HERS certification focused more on new construction. However, more recently that line has been blurred.

There are still some differences between the two programs, although they are more subtle. For example, BPI places strong emphasis on combustion analysis, while RESNET has recently focused on adopting standards for infrared analysis. In addition, HERS Raters are trained to use software that will provide a house with an overall efficiency rating. RESNET operates a quality assurance system for all HERS Raters.

Nonetheless, because the fundamental concepts in both types of training are mostly the same, it is worth examining the curriculum of whichever course you choose to make sure it includes the items you wish to learn. One reason to choose a particular certification may also be that it is required by a particular incentive program that you can tap into when marketing your services to potential customers.

\section{Article Snippets}

Home Energy Magazine:

\section{So You Want to Be a Home Performance Contractor?}

"Treating a home from a whole-house perspective means taking control of the home's flows of energy, heat, air, and moisture, and producing an indoor environment that will truly satisfy customers and turn them into the best advertising any contractor could have: happy customers who are excited to tell others what you did to their homes." 4/16/2006.

http://www.homeenergy.org/show/article/nav/archive/page/7/id/199

\section{Adding Home Performance Contracting to an Existing $\underline{\text { HVAC Business }}$}

"We at AirRite Air Conditioning Company, Incorporated, were no different from other contractors! We attended manufacturers' training seminars, trying to stay up-to-date on load calculations, duct designs, infiltration testing, house sealing, and values and types of insulation. Even with this new knowledge, though, we found that customers still had problems with uneven temperatures, poor air quality, dusty homes, and combustion safety. We kept wondering why...

Home performance contracting has since opened our eyes and minds to new sources of revenue for our company. 
We started to see that sealing recessed can lights, sealing ducts, and sealing thermal bypasses were all possible new revenue streams, as were installing dehumidifiers, heat recovery ventilators, humidifiers, attic ventilation, and attic insulation. Many other possibilities were available if we just opened our eyes." 4/16/2006

http://www.homeenergy.org/show/article/nav//id/206

\section{Resources}

Home Energy Magazine Archive:

http://www.homeenergy.org/

BPI Training:

http://www.bpi.org/schedules training.aspx

RESNET HERS Rater Training:

http://www.resnet.us/programs/training

RESNET Mortgage Industry National Home Energy Rating Standards:

http://www.resnet.us/standards/mortgage

"Air Sealing and Insulation That Works, ENERGY STAR. http://www.energystar.gov/index.cfm?c=behind the walls. btw airsealing 


\section{Appendix H - Customer Presentation Process}




\section{HVAC Transition Guide for Building Performance Contractors}

\section{Operational Area: Sales}

\section{Work Activity: Customer Presentation Process}

\section{Issue}

The customer presentation process is key to selling home performance improvements. The more the customer understands about how their house works, what is wrong with it, what you can do to fix it, and the potential benefits, the greater the likelihood that you will sell the job.

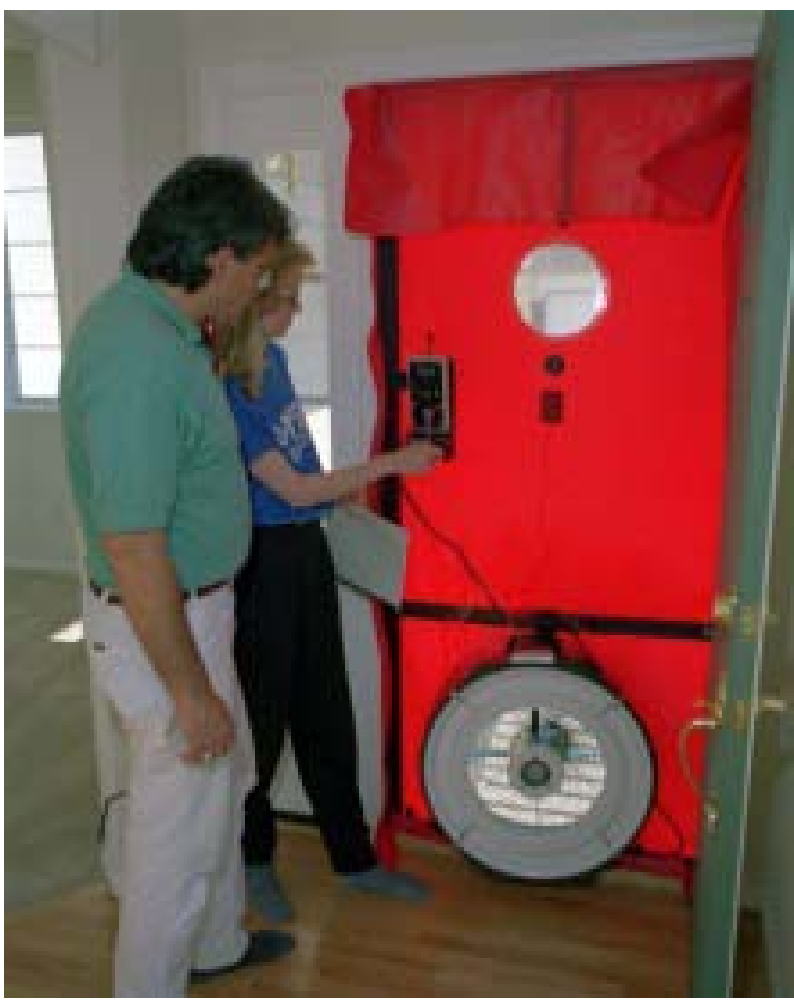

(National Renewable Energy Laboratory/PIX 04895,

http://www.nrel.gov/data/pix/Jpegs/04895.jpg)

\section{Best Practices}

The presentation process is all about gaining the customer's trust so they will believe your results and buy into your recommendations for installation. Letting the customer get involved can be an effective way of getting them on board. Short of that, you should make your presentation as clear as possible and back it up with pictures and graphics that are easy to interpret. Some additional customer presentation tips include the following:

- Invite the homeowner to tag along and assist when you are conducting the home assessment and diagnostic tests. Letting the customer see what you are doing will give them more confidence in the assessment results you present to them.

- Take lots of pictures of deficiencies and retrofit opportunities in the house, and show the owner as part of your presentation process. This way the customer can actually see where there are problems and will not simply have to "take your word for it."

- Present pros and cons and good-better-best options every time while clearly explaining the differences. Make sure the customer understands their home performance issues and the possible solutions. Encourage them to ask questions.

- Consider using a laptop to show the customer your results in an interactive way using modeling software after you have performed your assessment. Let the customer participate in the process of evaluating the status quo versus potential installation option. A customer who has become involved in this way is more likely to be committed to the improvements because they know where the projected benefits come from.

- Explain the full range of benefits of your proposed improvements. Present cost savings in terms of "rate of return," comparing the results to what a customer's $401(k)$ or other investments might be getting. Highlight the health, safety, and environmental benefits that the measures will create as well.

- Bring a portable printer with you, and print out the results of your assessment, as well as the installation plan. This way there will be no questions later about the work to be done.

\section{Modeling Software}

Once you have conducted your whole house assessment, your results will become the inputs in your energy modeling software, which in turn serves as the basis for the customer presentation. There is a wide range of software programs on the market, so it is worth exploring different options. The key is to make sure the software you choose is not only accurate but also presents results in a way that can be easily interpreted. Follow these tips for optimal use of modeling software: 
- Strive for the highest level of accuracy of predictions you can afford to ensure believability. Consider software that can adjust these predictions based on the customers' actual utility bills. Some new tools achieve impressive levels of accuracy with minimal inputs.

- Ensure that the presentation of the results is in a graphic-rich, customer-friendly format. Print out the results, and leave them with the customer if possible. Spend the time necessary to educate the customer on the report so that they can explain it to their spouse.

- Provide a rating or label for the home, both as is and after recommended improvements. This simplified quantification can serve as a powerful means of boiling down lots of details into a single understandable metric.

The cost of energy analysis software ranges widely from a few hundred dollars to thousands. If you are fortunate enough to operate in an area that offers a Home Performance Program through your local utility, many times the software is made available at little or no charge.

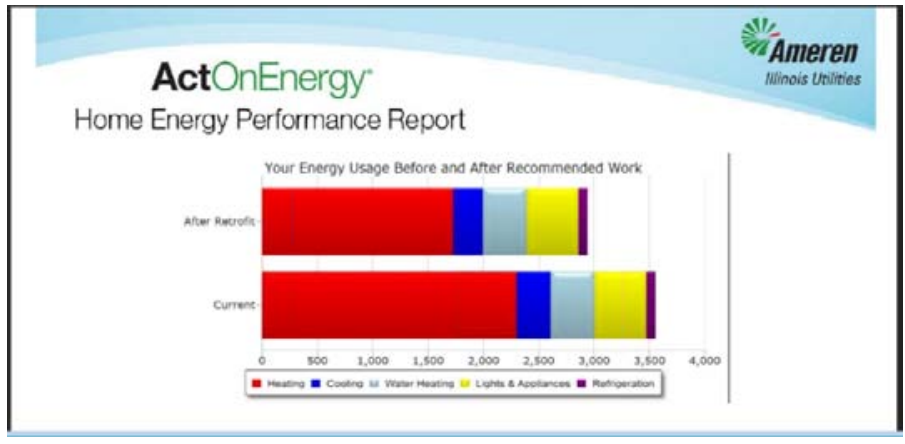

(Ameren Illinois Utilities,

http://www.ameren.com/sites/aiu/Pages/Home.aspx)

\section{Article Snippets}

\section{ACCA Contractor Excellence:}

\section{Contractor Takes Holistic Approach}

So the TAG team [TAG Mechanical, Syracuse, New York] attempts to educate their clients, effectively laying out the pros and cons of system alterations and installs. "We want our clients to be informed," [Ellis] Guiles, [vice president of TAG] said. "No matter what the decision of a client is, they will have everything laid in front of them, so they can make the best decision for them personally at that time." 9/20/2010.

http://www.contractorexcellence.com/2817

\section{Help Customers Choose High Efficiency}

Greg Gill, president and CEO, Action Air Conditioning, Heating, and Solar, San Marcos, California:

- "Sell customers whatever they want, but give everyone the same best-better-good presentation. Give everyone the same treatment the same way every time. Never assume someone can't afford a high-end system."

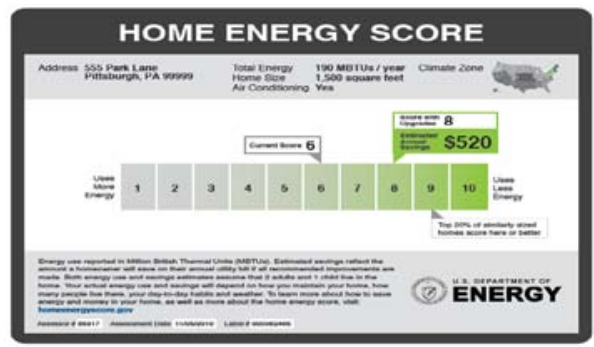

(U.S. Department of Energy,

http://www1.eere.energy.gov/buildings/homeenergyscore/index.html)

- "Take the time to show them the benefits of highefficiency equipment, including the return on investment."

- "Customers are often amazed by what they see in the pictures - crushed ductwork, holes in the plenum. They've never seen this before because most contractors don't bother going into the attic or crawl space to look at the whole system. When we take the time to look everywhere and show them what's wrong with their system, we find they are much more willing to spend the money with our company, even though we may be $\$ 3,000$ or $\$ 4,000$ higher than someone else." 7/6/2010 http://www.contractorexcellence.com/1940

\section{Tips for Selling Green}

Larry Taylor, president, AirRite Air Conditioning Co., Fort Worth, Texas:

- "We explain that the whole house is a puzzle, and if you leave a piece out, you end up with a hole in the middle of the picture. We actually use a picture of a puzzle with a piece missing when we talk with customers."

- "The main thing is to show the customer the results of the audit in a consumer-friendly way and then detail the process for completion of the project. After going through the reports with the customer and explaining the technical data, most will say, 'What is it going to take to fix my problems, and can you do it for me?' 
Wow, I hate those high-pressure sales calls!" 1/3/2011 http://www.contractorexcellence.com/3911

\section{Contracting Business}

What Every Consumer Really Wants to Know

Rob "Doc" Falke, president of National Comfort Institute:

"'Nothing happens 'til somebody sells something.' That's a common phrase that rings through sheet metal shops across the country, but in recent years our industry has learned that sales don't happen until we have 'taught the customer something."'

2/3/2010http://contractingbusiness.com/service/content/sales techniqu es0203/

\section{Resources}

Energy Star Financial Evaluation Calculators:

http://www.energystar.gov/index.cfm?c=assess value.fina ncial tools

U.S. Department of Energy's Building Energy Software Tools Directory:

http://apps1.eere.energy.gov/buildings/tools directory/subj ects sub.cfm

Home Energy Magazine:

http://www.homeenergy.org/list/archive

ACCA Contractor Excellence:

http://www.contractorexcellence.com/

Ameren Illinois Utilities:

http://www.ameren.com/sites/aiu/Pages/Home.aspx

U.S. Department of Energy, Home Energy Score http://www1.eere.energy.gov/buildings/homeenergyscore/i $\underline{\text { ndex.html }}$ 


\section{Appendix I - Subcontractor and Vendor Relations}




\section{HVAC Transition Guide for Building Performance Contractors}

\section{Operational Area: Business Planning/Process and Production}

\section{Work Activity: Vendor Relations and Subcontractor Relations}

\section{Issue}

Home performance contractors are dependent on a large network of partnerships that contribute in important ways to the completion of comprehensive home improvements. Vendors are essential in providing the necessary systems, appliances, and materials that will need to be installed, while subcontractors will often perform the actual installations. Establishing strong relationships on both sides of this supply chain will help you complete quality work in a timely and cost-effective manner, which in turn will contribute to your good reputation.

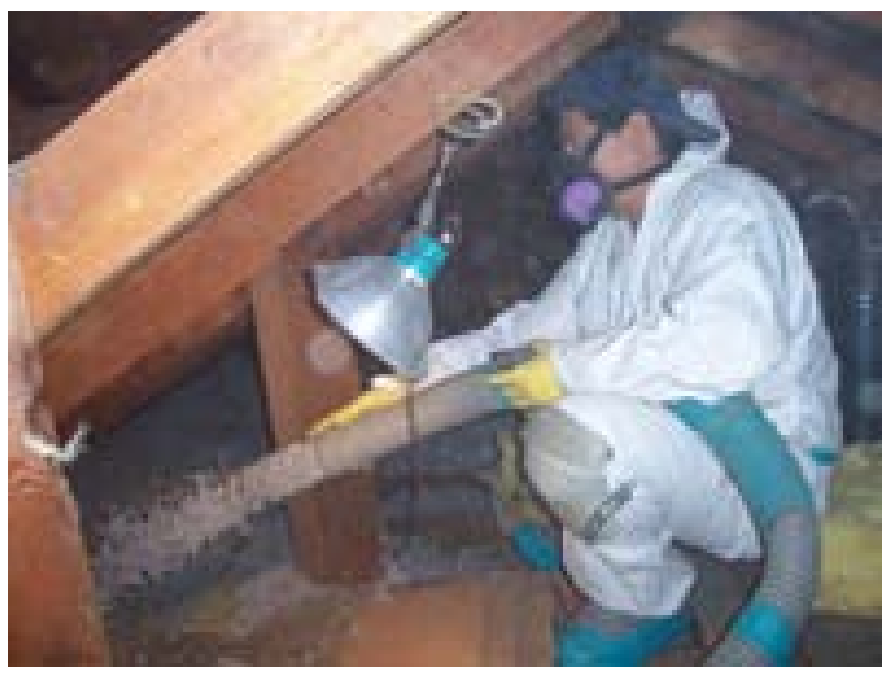

(U.S. Environmental Protection Agency,

http://www.epa.gov/region9/mediacenter/richmond/rb-hires.html)

\section{Vendors - Best Practices}

There are a number of factors to look for when choosing vendors to supply the materials for your home performance jobs. Cost is one factor, but other considerations are important as well. Keep in mind the following issues when evaluating which vendors to work with:

- $\quad$ Price versus quality. While a low price will help you make competitive bids, in the long run your reputation can be more impacted by the vendor's service, reliability, and quality. Find vendors who balance all of these factors well.

- Technical support. For complex systems, you may need vendors with the technical expertise and quality of service to provide guidance both during and after installation.

- Find vendors--or work to get your preferred vendor--to stock the best products for your home performance jobs. Don't necessarily trust that what they have always carried will suffice. Research the best home performance products (e.g., spray foam kits, duct mastic, caulking, HVAC equipment), and make sure your vendors keep these in stock.

- Loyalty versus shopping around. Sticking with one or just a few vendors can increase your quality of service and sometimes bring volume discounts. On the other hand, spreading out your orders allows you to search for lower prices and more easily meet designer, program, or customer specifications. Choose the option that works best for your business.

Once you have chosen your vendors, follow these guidelines to help you build and maintain productive relationships that will keep your interactions flowing smoothly:

- With every transaction, fill out a clearly written purchase order that specifies exactly what you expect to have delivered and when.

- Before completing your supply order, make sure you have established delivery and invoicing procedures that both parties agree upon.

- Try to avoid last-minute/expedited orders and frequent order changes to increase the chances of getting the correct supplies on time and on budget.

- Consider signing written agreements with key vendors that clarify the basic processes of your interactions and make them routine.

\section{Subcontractors - Best Practices}

Given the wide range of measures that often need to be installed as part of comprehensive home performance improvements, you may find yourself lacking certain types of expertise. This raises the question of whether you should try to develop the required knowledge in-house or partner with subcontractors for some of the work. Factors to consider when making this decision include the following: 
- Work flow. Hiring subcontractors may make sense for jobs that tend to be intermittent and unpredictable. Keeping employees on hand for this type of work may not be worth the cost.

- Quality control. Keeping your work in-house gives you direct oversight of all work that is done and can cut down on conflicts and complications. On the other hand, the benefits may not be worth it if a subcontractor with a good reputation can provide greater technical expertise.

- Timeliness. Subcontractors maintain their own schedules and may run into scheduling conflicts or be unavailable for a particular job. However, the flexibility of hiring subcontractors only when needed can still be cost effective.

Assuming you do choose to use subcontractors in at least some cases, the following tips will help you build and maintain productive relationships with them:

- Require contractors to demonstrate proper licensing and other credentials and provide references that establish their capacity to perform competent, quality work.

- Be sure that all subcontractors carry proper insurance and workers' compensation policies. Your own provider can advise you on whether a subcontractor's policies should name you as an additional insured for a given job.

- Set a timeline for job completion, and establish clear milestones that will keep everyone on schedule.

- Perform regular inspections of your subcontractors' work to correct any problems before they become too large.

- Set clear expectations about the level of quality you require, including conditions for termination based on underperformance and incentives for exceptional work.

- Be sure to follow federal guidelines that define a subcontractor versus an employee. Consult with your legal advisor to be sure you do not cross this line unintentionally.

\section{Article Snippets}

RB Lewis Associates:

A Way to Build Great and Lasting Vendor Relationships

- "Plumbing the depths for the low cost vendor when motivated solely by greed often ends up badly. Is your strategy based on how much money you can save by attracting the cheapest seller? How would you like it if your customer admitted they hired you only because you were the cheapest and your value proposition or quality reputation had no bearing on the buyer's decision - that they were hiring you solely to maximize their margin?"

- "Look first internally when you are finally ready to implement your vendor relations strategy. Create the right conditions for strong supplier performance. Collaborate with your suppliers, and develop their scope of services consistent with each other's identified needs. Articulate clearly defined responsibilities, yours and theirs. Perform to high standards, and demand they do, too." 12/2010 http://rblewisassociates.com/wp-content/uploads/2011/01/vendorrelations-strategy.pdf

Home Energy Magazine:

So You Want to Be a Home Performance Contractor

"General contracting works if you can keep down your cost of subs and if jobs are intermittent and you don't want to try to carry employees over the slow periods. But one of the basic tenets of home performance contracting is doing quality work. Getting quality insulation and HVAC subcontractors to work for you for below-market rates may not be easy. This can work if you have the marketing skills that the subcontractors lack and can keep them busy and happy." 4/16/2006

http://www.homeenergy.org/show/article/nav/archive/page/7/id/199

\section{Lowe's Commercial Services:}

Subcontractors vs, Full-Time Employees: What's Best for Your Company?

"If you stock your contacts list with quality subs, you'll have the flexibility of inserting them on a project without the year-round financial burden of a full-time staffer. Many contractors use subs in order to save money, because they can pre-determine the cost of a project regardless of how many hours it will take. Keep in mind that someone who is juggling multiple jobs may not always be available when you need him. Therefore, if you plan to use the same subs on a regular basis, try to put the same consideration into who you hire as you would with an employee." 3/2011

http://www.lowesforpros.com/subcontractors-vs-full-time-employeeswhats-best-for-your-company 
Deep-Energy Retrofits for Existing Homes

"[Don Ferrier, president of Ferrier Custom Homes and Ferrier Builders, Inc. in Fort Worth, Texas] recommends setting up full screenings of your subcontractors where the conversation is thorough and outlines exactly what a retrofit will entail and what is expected of the subcontractor, including questions of how they plan to implement and perform their work. Then, you can determine whether or not the subcontractor is right for the job." $5 / 2010$

http://www.lowesforpros.com/deep-energy-retrofits-for-existing-homes

\section{Resources}

RB Lewis Associates:

http://rblewisassociates.com

Home Energy Magazine:

http://www.homeenergy.org/list/archive

Lowe's Commercial Services:

http://www.lowesforpros.com

"Federal Stimulus Dollars Help Train Disadvantaged Richmond Residents for Jobs in Renewable, Clean Energy," U.S. Environmental Protection Agency. http://www.epa.gov/region9/mediacenter/richmond/rb$\underline{\text { hires.html }}$ 


\section{Appendix J - Full Value Ratings of Work Activities}




\section{Appendix $\mathbf{J}$ - Full Value Ratings of Work Activities}

\begin{tabular}{|c|c|c|c|c|}
\hline Operational Area & Work Activity & $\begin{array}{l}\text { Value of } \\
\text { Guidance to } \\
\text { Contractor }\end{array}$ & Difficulty & Sum \\
\hline $\begin{array}{l}\text { Business Planning/ } \\
\text { Process }\end{array}$ & Training & 4 & 2 & 6 \\
\hline $\begin{array}{l}\text { Business Planning/ } \\
\text { Process }\end{array}$ & Manufacturer Relations & 1 & 1 & 2 \\
\hline $\begin{array}{l}\text { Business Planning/ } \\
\text { Process }\end{array}$ & Equipment & 4 & 3 & 7 \\
\hline $\begin{array}{l}\text { Business Planning/ } \\
\text { Process }\end{array}$ & $\begin{array}{l}\text { Equipment } \\
\text { Maintenance/Calibration }\end{array}$ & 2 & 2 & 4 \\
\hline $\begin{array}{l}\text { Business Planning/ } \\
\text { Process }\end{array}$ & Vendor Relations & 4 & 4 & 8 \\
\hline $\begin{array}{l}\text { Business Planning/ } \\
\text { Process }\end{array}$ & Strategic Planning & 4 & 4 & 8 \\
\hline $\begin{array}{l}\text { Marketing/ } \\
\text { Customer Contact }\end{array}$ & Customer Education & 4 & 4 & 8 \\
\hline $\begin{array}{l}\text { Marketing/ } \\
\text { Customer Contact }\end{array}$ & Community Engagement & 4 & 4 & 8 \\
\hline $\begin{array}{l}\text { Marketing/ } \\
\text { Customer Contact }\end{array}$ & Social Media & 1 & 1 & 2 \\
\hline $\begin{array}{l}\text { Marketing/ } \\
\text { Customer Contact }\end{array}$ & Direct Referral Programs & 2 & 1 & 3 \\
\hline $\begin{array}{l}\text { Marketing/ } \\
\text { Customer Contact }\end{array}$ & Newsletter & 1 & 1 & 2 \\
\hline $\begin{array}{l}\text { Marketing/ } \\
\text { Customer Contact }\end{array}$ & Service Tech Referrals & 2 & 1 & 3 \\
\hline $\begin{array}{l}\text { Marketing/ } \\
\text { Customer Contact }\end{array}$ & Call Scripting & 2 & 1 & 3 \\
\hline $\begin{array}{l}\text { Marketing/ } \\
\text { Customer Contact }\end{array}$ & Inbound Call Process & 2 & 1 & 3 \\
\hline $\begin{array}{l}\text { Marketing/ } \\
\text { Customer Contact }\end{array}$ & Recurring Call Schedule & 0 & 0 & 0 \\
\hline Assessment & $\begin{array}{l}\text { Pre-Assessment } \\
\text { Preparation }\end{array}$ & 3 & 1 & 4 \\
\hline Assessment & Arrival Process & 4 & 3 & 7 \\
\hline Assessment & On-Site Customer Interview & 4 & 4 & 8 \\
\hline Assessment & $\begin{array}{l}\text { Whole House Performance } \\
\text { Assessment }\end{array}$ & 4 & 4 & 8 \\
\hline Sales & $\begin{array}{l}\text { Customer Presentation } \\
\text { Process }\end{array}$ & 4 & 3 & 7 \\
\hline Sales & Assessment Report & 4 & 3 & 7 \\
\hline Sales & $\begin{array}{l}\text { Phased Implementation } \\
\text { Sales Process }\end{array}$ & 3 & 3 & 6 \\
\hline
\end{tabular}




\begin{tabular}{|c|c|c|c|c|}
\hline Operational Area & Work Activity & $\begin{array}{l}\text { Value of } \\
\text { Guidance to } \\
\text { Contractor }\end{array}$ & Difficulty & Sum \\
\hline Sales & Rebates and Incentives & 1 & 1 & 2 \\
\hline Contract Administration & Financing Mechanisms & 1 & 3 & 4 \\
\hline Contract Administration & Loan Processing & 1 & 1 & 2 \\
\hline Contract Administration & $\begin{array}{l}\text { State or Local Program } \\
\text { Reporting }\end{array}$ & 4 & 4 & 8 \\
\hline Production & Work Scope Procedures & 4 & 4 & 8 \\
\hline Production & $\begin{array}{l}\text { Customer Interaction } \\
\text { Procedure }\end{array}$ & 3 & 2 & 5 \\
\hline Production & Job Completion/Test-Out & 3 & 3 & 6 \\
\hline Production & Job Completion Verification & 4 & 1 & 5 \\
\hline Production & $\begin{array}{l}\text { Post-Installation } \\
\text { Verification Sample }\end{array}$ & 1 & 1 & 2 \\
\hline Production & $\begin{array}{l}\text { Sponsoring Program } \\
\text { Quality Assurance }\end{array}$ & 1 & 1 & 2 \\
\hline Production & Subcontractors & 4 & 3 & 7 \\
\hline Customer Service & Diagnostics & 2 & 3 & 5 \\
\hline Customer Service & Repair/Replacement & 2 & 3 & 5 \\
\hline
\end{tabular}




\section{U.S. DEPARTMENT OF Energy Efficiency \& ENERCY Renewable Energy}

